\title{
Somatic cell-derived organoids as prototypes of human epithelial tissues and diseases
}

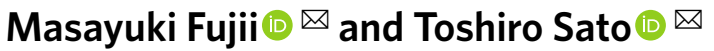

\begin{abstract}
Recent progress in our understanding of the regulation of epithelial tissue stem cells has allowed us to exploit their abilities and instruct them to self-organize into tissue-mimicking structures, so-called organoids. Organoids preserve the molecular, structural and functional characteristics of their tissues of origin, thus providing an attractive opportunity to study the biology of human tissues in health and disease. In parallel to deriving organoids from yet-uncultured epithelial tissues, the field is devoting a growing amount of effort to model human diseases using organoids. This Review describes multidisciplinary approaches for creating organoid models of human genetic, neoplastic, immunological and infectious diseases, and details how they have contributed to our understanding of disease biology. We further highlight the potential role as well as limitations of organoids in clinical practice and showcase the latest achievements and approaches for tuning the organoid culture system to position organoids in biologically defined settings and to grant organoids with better representation of human tissues.
\end{abstract}

$\mathrm{H}$ uman and other multicellular organisms harness the selfrenewal and multidifferentiation capacities of somatic stem cells as the main engine for sustaining tissue homeostasis. In particular tissues, progenitor or quiescent cells restore their stem cell programme upon tissue damage to fuel regeneration and safeguard tissue integrity. Disorderly stem cell self-renewal and regeneration may trigger tumourigenesis or tissue degeneration. A wealth of the understanding of signalling pathways and microenvironmental regulations that orchestrate these homeostatic, regenerative and pathologic conditions in epithelial tissues has allowed us to instruct somatic stem cells that are isolated from their home tissue to self-renew ex vivo and organize into tissue-resembling three-dimensional (3D) structures, or organoids, in a serum- and feeder-cell-free setting. With relentless efforts to devise culture recipes on an individual tissue basis, organoid technology now covers a number of human epithelial tissues with a healthy or diseased background. As the organoid research nears to the tentative goal of enabling a variety of somatic stem cells to develop into $3 \mathrm{D}$ tissues on a culture dish, spin-off studies have focused on the biological relevance of normal and disease organoids to real-world human tissues and explored how the groundbreaking technology can carve out a niche in basic science and biomedical research.

In this Review, we outline the fundamentals and the practical usage of organoids through the lens of tissue and disease modelling, and discuss how organoids rival and outperform traditional cell line models in current experimental and preclinical application. We further describe multilateral strategies in building human disease mimicries using patient-derived organoids and cutting-edge applications, including genome-editing technology and hybrid culture systems. Efforts to refine pre-existing organoid culture environments and to create more tissue-relevant organoids are renovating the organoid culture platform towards enhanced capabilities in the laboratory and the prospective use of organoid technology at the bedside. These movements to broaden the application of organoids has transformed the shape of human tissue research and may eventually usher in a next-generation biomedical platform that enables enhanced in vitro representation of human tissues and aids detection, diagnosis, prognostication and cure of human diseases in a personalized fashion.
Previous work has utilized the term 'organoid ${ }^{1,2}$ or coined analogue terms such as 'enteroids' or 'tumouroids ${ }^{33}$ independently and perhaps arbitrarily, leading to a dearth of a solid definition and nomenclature for in-vitro-cultured organotypic structures. To avoid confusion, we here define organoids as any heterotypic structures that can be reproducibly generated from single cells or cell clusters derived from somatic tissues or pluripotent stem cells, can self-assemble through cell-cell and cell-extracellular matrix (ECM) communications, and have some features of counterpart in vivo tissues. Besides adult somatic cells, organoids can also be created from pluripotent stem cells by directed differentiation in this paradigm. The application of pluripotent-stem-cell-derived organoids has been covered extensively by others and organoids hereafter refer to adult tissue-derived organoids unless otherwise specified.

\section{The organoid culture system}

The keystone of organoid technology is the ability of isolated somatic epithelial stem cells to reconstitute the structure and function of the tissue of origin when they are deposited in an appropriate context or the 'niche.' Organoid culture systems that direct the stem cells to manifest this ability can therefore be epitomized as the fabrication of the stem cell niche $e^{4}$. The initial derivation of mouse small intestinal organoids has verified this concept in a tangible form ${ }^{5}$, and the ensuing efforts to culture other tissues as organoids have basically inherited a similar culture style. An orthodox protocol for organoid establishment and culture first liberates tissue stem cells or stem-cell-containing units, for instance, intestinal crypts $^{5}$, prostate acini $^{6}$ and mammary glands ${ }^{7}$, from the underlying mesenchyme by physical and enzymatic isolation (Fig. 1). While particular tissues, such as the adult liver and pancreas, show sparse sign of tissue turnover in homeostasis, they still can rejuvenate the self-renewal activity in dormant cells, for example, pancreatic duct cells ${ }^{8,9}$, intrahepatic duct cells ${ }^{10,11}$ and hepatocytes ${ }^{12,13}$, upon specific stimuli, including inflammation, tissue injury and tumourigenesis. Organoid culture enables an expansion of such cells that show conditional proliferation by providing key signals that coax them out of dormancy. The stem cells or tissue fragments are then mounted into the ECM scaffold and overlaid with culture medium supplemented with combinations 
a

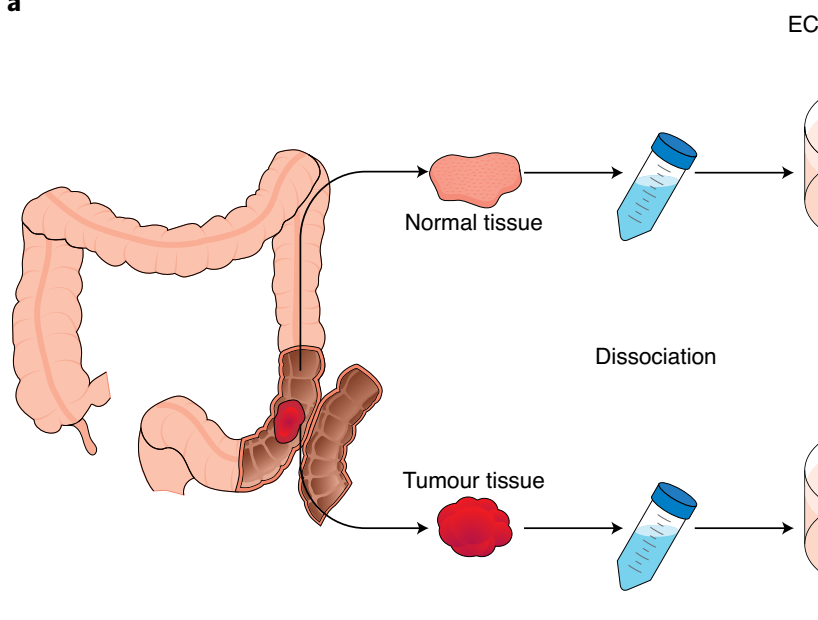

$\mathrm{ECM}-\quad-$ Culture medium with stem cell niche factors
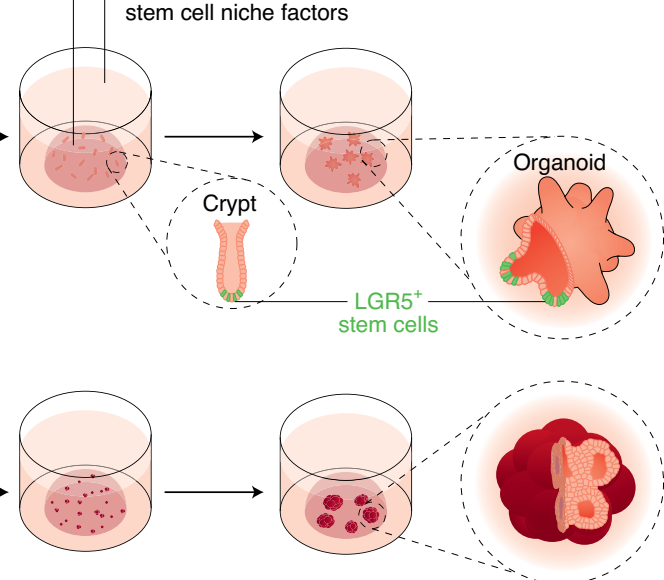

b

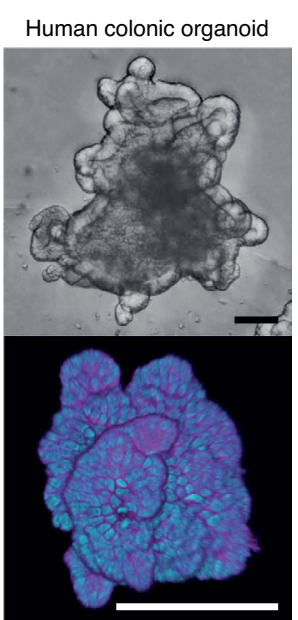

Fig. 1 | The organoid culture system. a, An example of organoid derivation from human normal colonic epithelium and a colon tumour. Isolated epithelial stem cells or stem cell-containing units (crypts) are embedded into the ECM and nourished with culture medium that includes essential niche factors. Within a week, the stem cells self-organize into organoids with a single lumen and multiple buds. Tumour organoids can be cultured in a similar way and usually form structures that represent the original histology. b, Representative bright-field (left) and 3D-reconstructed fluorescence (right) images of a human normal colon organoid. Plasma membrane (magenta) was stained with CellMask Plasma Membrane (Thermo Fisher Scientific) and nuclei (cyan) were counterstained with Hoechst 33342. The fluorescence image was captured by FVMPE-RS multiphoton microscope (Olympus). Scale bar, $100 \mu$ m.

of mitogens, morphogens and cytokines that guide the self-renewal and organized differentiation of somatic stem cells. These stem cell niche factors are typically selected from the activators of the canonical Wnt pathway (Wnt-3a and R-spondin) and the mitogen-activated protein kinase (MAPK) pathway (epidermal growth factor (EGF) and fibroblast growth factor (FGF)), and the inhibitors of the bone morphogenetic protein (BMP) pathway (Noggin and Gremlin) and the transforming growth factor- $\beta$ (TGF- $\beta$ ) pathway (activin receptor-like kinase inhibitor). Unfortunately, no one-size-fits-all solution that allows organoid expansion regardless of the tissue origin has been crafted owing to the substantive tissue-to-tissue variability of the dependency on the stem cell niche. It is also challenging to determine a priori the biological activity of individual niche factors in a target tissue. Previous studies have therefore configured tissue-specific culture conditions by nominating potentially active niche factors, followed by individual assessment of their impact on organoid growth and long-term culture sustainability. This down-to-earth strategy has so far been prolific and has allowed us to culture various murine epithelial tissues ${ }^{14}$ and, shortly thereafter, human normal and diseased epithelial tissues ${ }^{6,15-36}$ as organoids (Fig. 2 and Table 1). Organoid derivation from other domesticated and laboratory animals, including pigs, dogs, cats and rats, have also been described ${ }^{24,37-39}$, and a similar culture setting further allowed an organoid expansion of snake venom glands ${ }^{40}$, underscoring the cross-species versatility of the organoid culture system. It should be noted that the growth factor combinations are not only tailored to individual tissues but also include unique molecules in some cases, such as gastrin for digestive tissues, neuregulin-1 for mammary organoids ${ }^{7,18,19}$, dihydrotestosterone for prostate organoidsd ${ }^{6,41}$ and forskolin for human liver bile duct organoids ${ }^{25}$ but not for hepatocyte organoids ${ }^{26}$. Differing growth factor conditions also exist for some tissues, suggesting a margin for further optimization.

Unlike primary cells that are considered to have finite replication capacity and eventually enter senescence (the Hayflick limit), many organoids can undergo stable expansion with serial passaging for over one year when placed under optimal culture conditions, reflecting the ability of particular tissue stem cells to self-renew throughout a lifetime. This capability of organoid culture may partially be attributed to the activation of telomerase in stem cells via enhanced Wnt signalling ${ }^{42,43}$, given the vital role of telomere capping in ensuring sustained cell replication and telomerase activation in murine intestinal stem cells ${ }^{44}$ and hepatocytes with regenerative potential ${ }^{13}$. Nevertheless, some organoids, including human oesophagus $^{20,21}$ and human hepatocyte ${ }^{26}$ organoids, cease proliferation after a few months of culture. Whether the growth arrest in such cases results from cell-inherent growth limitation or the insufficiency of the culture composition remains largely unknown. Also, because the organoid culture systems that we highlight here are dedicated to specifically expand the epithelial lining of given tissues, they do not support the stable maintenance of non-epithelial components, including connective tissues, vessels and nerve. Deriving organoids from organs that that are mainly constituted of such non-epithelial elements, such as the heart and brain, may thus require completely different strategies.

In organoid culture, tissue stem cells self-renew and together with their progenies self-organize into 3D clusters that show structural, functional and molecular similarity to the tissue of origin. For example, organoids derived from human small intestinal epithelium assume a monolayered cystic morphology with crypt-like protrusions that lodge leucin-rich repeat-containing G-protein receptor 5 (LGR5)-positive stem cells at their summits ${ }^{23,24}$. Human endometrium-derived organoids generate glandular structures that retain an expression of endometrium marker proteins and respond to female hormones ${ }^{33,34}$. Such biological conformity of organoids to their counterpart tissues has been the driving force of the rapid dissemination of organoid technology and the usage of organoids in studying human epithelial tissues in a physiologically relevant setting. Besides the standardized culture procedures, the organoid platform is receptive to the modification of its three components, the organoids, ECM and culture medium condition. As discussed later in detail, recent studies have focused on tuning and controlling structural, compositional and biochemical properties of each of these elements to extend basic and clinical application of organoids.

\section{Comparison with other human cytological models}

Aside from the recent cascade of successful organoid derivation from diverse human tissues, a plethora of human cell lines established so far have served as the main substitute of living human 


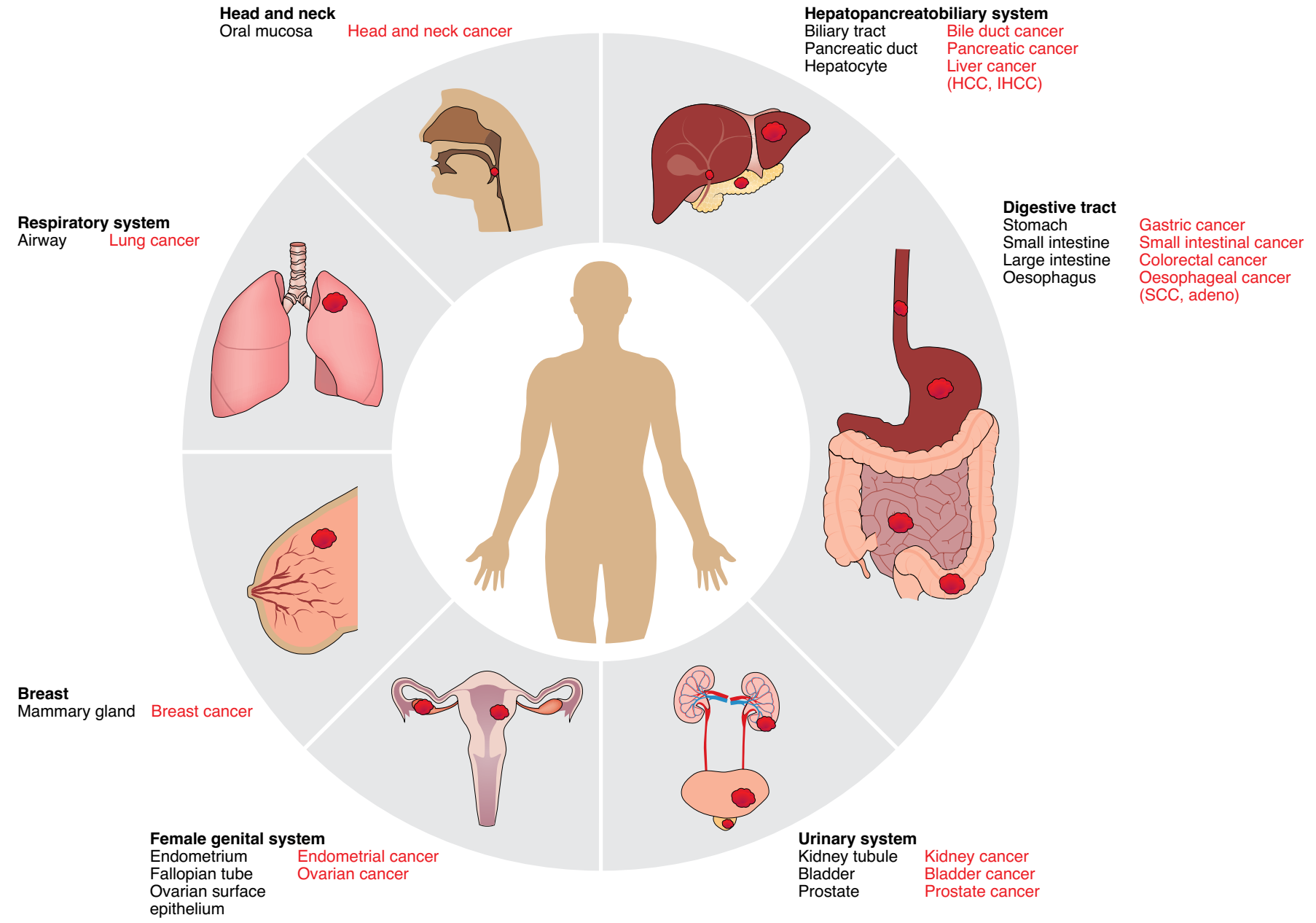

Fig. 2 | Range of human tissues in organoid technologies. Human epithelial tissues that are expandable as organoids are shown. Normal and tumour tissues from which organoids have been derived are shown in black and red text, respectively. Origins of tumour-derived organoids are also presented in red. HCC, hepatocellular carcinoma; IHCC, intrahepatic cholangiocarcinoma; SCC, squamous cell carcinoma; adeno, adenocarcinoma.

tissues. Organoid and cell line cultures share commonality with respect to their ability to expand human tissue cells, yet these models differ in multiple dimensions including their topology, cellular composition and relevance to parental tissues (Fig. 3).

Human cell line culture in general adopts an adherent monolayer system using planar plastic substrates and an overlay with serum-supplemented media. While this format offers simplicity and high throughput, its deviation from the natural tissue environment can cast a bottleneck in the derivation of primary cell lines; the majority of cells that lack fitness drop out upon being transferred to a non-permissive culture environment. In addition, the poor compositional transparency and batch-to-batch variability of the serum, which is essential for many cell lines as a growth factor substitute, can complicate the interpretation of cell line behaviours. A detrimental effect of the serum on the growth of pancreatic ductal $^{30}$ and liver ductal ${ }^{25}$ organoids has also been documented. For these reasons, cell line derivation is often interrupted by the low establishment efficiency, which does not exceed $30 \%{ }^{45}$, and successful cell line propagation may represent the survivorship of minor privileged cells that can accommodate themselves to adverse conditions. Most of the authenticated human cell lines are indeed of cancer or embryonic cell origin, and normal epithelium-derived cell lines are immortalized by virus transformation. Cancer cell lines are yet filtered by the strong selection bias, and the majority of them derive from high-grade tumours. They therefore show poor differentiation as xenograft tumours and do not represent the histological and molecular diversity of human cancers ${ }^{46,47}$. Furthermore, unlike the columnar or (pseudo) stratified cell organization and heterogeneous cellular assembly in tissues and 3D organoids, adherent cell lines assume a flat morphology with minimal cell-to-cell variation. Some cell lines can form 3D clusters or spheres in suspension or soft agar, but these structures still have uniform cellularity and are rather used for semi-quantitative inference of anchorage-independent growth capacity and clonogenicity.

These dissimilarities between organoids and cell lines point to differential arenas where these models can leverage their strengths. Tissue-relevant organoids are a preferable model for studying the biology and physiology of human tissues in health and disease, as well as for the potential usage in clinical practice such as precision and regenerative medicine. However, cell lines lend themselves to other fields such as cellular biochemistry research to investigate molecule interactions and modifications. Biologically homogenous and scalable cell lines can dedicate themselves to supply specialized culture by-products, for instance, as the feeder for conditioned medium and for culture of other cell types. High-throughput genetic perturbation assays including large-scale knockout screening also favour cell lines owing to the uniform growth of cell lines. Thus, organoids and cell lines are distinct but not completely exclusive, and the appropriate usage of these models is highly subject to the purpose of the research. 


\section{Table 1 | Organoid models of normal human tissues}

Tissues

Growth factor supplements

Culture duration Refs.

Head and neck

Oral mucosa

R-spondin, CHIR99021, EGF, FGF-2, FGF-10, Noggin, forskolin, prostaglandine E2, A83-01, B27

Respiratory

Airway

R-spondin, FGF-7, FGF-10, Noggin, A83-01, SB202190, B27

Wnt-3a, EGF, FGF-2, Noggin, A83-01, N2, B27

$>1$ year

Breast

Mammary

R-spondin, EGF, FGF-7, FGF-10, neuregulin-1, Noggin, A83-01, SB202190

$>10$ passages

Digestive tract

Oesophagus

Wnt-3a, R-spondin, EGF, FGF-10, Noggin, Gastrin, A83-01

-20 passages

EGF, bovine pituitary extract

Stomach

Wnt-3a, R-spondin, EGF, FGF-10, Noggin, gastrin, A83-01

Small and large intestine

Wnt-3a, R-spondin, EGF, Noggin, gastrin, A83-01, SB202190

Wnt-3a, R-spondin, EGF, IGF-1, FGF-2, Noggin, gastrin, A83-01

4 passages

$\sim 3$ passages

$>1$ year

$>1$ year $^{\mathrm{a}}$

$>1$ year $^{\mathrm{a}}$

Hepatopancreatobiliary

Liver (ductal)

R-spondin, FGF-10, HGF, forskolin, gastrin, A83-01, N2, B27

Liver (hepatocyte)

R-spondin, CHIR99021, EGF, FGF-7, FGF-10, HGF, TGF-a, gastrin, A83-01, B27

$>6$ months

R-spondin, EGF, DKK-1, dexamethasone

R-sponin, EGF, FGF-10, HGF, Noggin, N2, B27

Pancreatic duct

Wnt-3a, R-spondin, EGF, Noggin, gastrin, A83-01

2-3 months

$>20$ passages

$>16$ weeks

$>6$ months $^{a}$

Genitourinary

Kidney tubule

R-spondin, EGF, FGF-10, A83-01,B27

$>6$ months

FGF-2, FGF-7, FGF-10, A83-01, B27

$>30$ passages

$>6$ months

R-spondin, EGF, FGF-10, HGF, Noggin, A83-01, N2, B27

Endometrium

Wnt-3a, R-spondin, EGF, FGF-10, Noggin, A83-01, N2, B27, SB21092, E2

$>4$ months

Wnt-3a, R-spondin, EGF, FGF-10, Noggin, SB431542, N2, B27

$>16$ months

Fallopian tube

Ovarian surface epithelium

Prostate

R-spondin, EGF, FGF-2, Noggin, dihydrotestosterone, prostaglandine E2, A83-01, SB202190, B27 >12 months

Growth factor components and culture duration in previously reported organoid culture systems. N2, N2 supplement; B27, B27 supplement. A83-01, CHIR99021 and SB202190 are inhibitors of TGF- $\beta$ receptor, GSK-3 and p38 MAPK, respectively. ${ }^{a}$ Confirmed in our laboratory.

\section{Organoids as disease models}

The stability and versatility of the organoid culture system have provided us with a unique window of opportunity to approach human diseases that affect epithelial tissues. Epithelium-autonomous dysregulation and extrinsic factors that misguide cellular behaviours can independently or interactively underlie such diseases. Therefore, organoid-based modelling of human diseases has basically focused on phenocopying either or both of these two disease facets depending on the disease nature. Epithelium-autonomous molecular aberrations, mainly genetic and epigenetic modifications, and cytological phenotypes such as clonogenic growth, histological abnormality and drug response are reconstituted by direct culture of disease tissues or by genetic engineering of organoids. Intercellular dialogue between epithelial and non-epithelial cells can be resumed ex vivo by co-culturing non-epithelial cells with epithelial organoids. Imitating pathogenic tissue environments by modulating culture conditions is a simple but powerful strategy to investigate the role of environmental regulation in disease phenotypes. The following sections describe practical operations of organoid-based disease modelling systems and how they contributed to our understanding of disease biology. In any cases, organoids derived from healthy tissues provide an important reference material for gauging the molecular and phenotypic abnormality of disease organoids.
Direct derivation of organoids from diseased tissues. The organoid culture condition optimized for a specific normal tissue consistently enables an expansion of diseased epithelium of the common tissue origin. This capability allowed us not only to handle a range of diseases as living tissue models but also to overcome experimental hurdles attributed to the limited availability of disease tissues. While explorative studies using human disease tissues have traditionally suffered from many reasons, such as the rarity of the subject disease, poor anatomical accessibility of the target tissue and ethical concerns, organoids that can be amplified even from minute tissues sampled by needle biopsy provide an ample resource of disease tissues that can directly be used for various downstream applications (Fig. 4a).

A representative example of patient-derived organoids is cancer organoids. In parallel with the extending coverage of human epithelial tissues by organoid technology, tumour-derived organoids now encompass miscellaneous neoplasms of epithelial origin, specifically carcinomas of gastrointestinal (oesophagus ${ }^{48,49}$, stomach $^{22,50-52}$, large intestine $e^{23,53-59}$, liver ${ }^{60-62}$, biliary $\operatorname{tract}^{63}$ and pancreas ${ }^{29,30,56,64,65}$ ), craniocervical $^{15,49}$, mammary ${ }^{18}$, genitourinary (endometrium ${ }^{33,66}$, ovary $^{36,67}$, bladder ${ }^{32,68}$, kidney ${ }^{56,69}$ and prostate ${ }^{41,70}$ ) and respiratory (lung ${ }^{16,17,56}$ ) systems. Although the establishment efficiency of tumour organoids is largely contingent on the sample volume, purity and origin, the derivation rate of organoids from fresh tumour tissues 


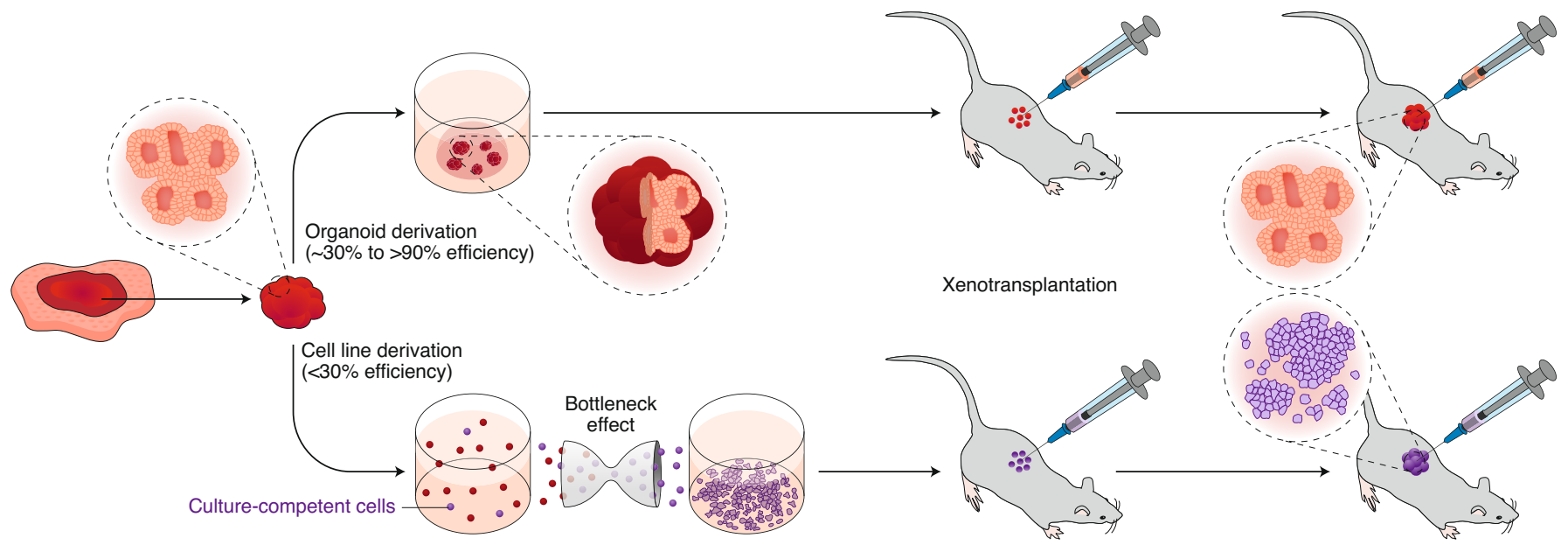

Fig. 3 Distinctions between organoids and cell lines. An illustrative example of derivation of organoids (top) and a cell line (bottom) from a tumour that shows moderate differentiation. Organoids preserve differentiated structures, such as glandular and cribriform structures, during culture and as xenograft tumours. In contrast, cell line culture only enables expansion of culture-proficient cells that usually form undifferentiated xenografts.

typically falls between $30 \%$ and over $90 \%$, far exceeding the probability of establishing stable cancer cell lines from the same tissues ${ }^{17,71}$. Omics-based comparisons between tumour-derived organoids and original tumour tissues demonstrated that tumour organoids carry over the molecular fingerprints of the original tumours. Tumour organoids under culture also morphologically echo the histological grade or differentiation status of the original tumours. This molecular and phenotypic credibility of tumour-derived organoids as the substitute for patient tumours has facilitated their usage in functional assays, including genetic perturbation experiments and drug screening on a per-patient basis. Efforts to comprehensively catalogue tumour organoids are beginning to archive the extensive diversity of the histological and molecular subtypes in human cancers. Tumour organoids grown from single tumour cell clones captured intratumour heterogeneity of genetic mutations and drug sensitivity at the clone level ${ }^{72}$. Aside from the immense possibilities of patient-derived tumour organoids, organoids with chromosomal instability ${ }^{73,74}$ or mismatch-repair deficiency ${ }^{54,75}$ continuously accrue chromosome mis-segregations or replication errors during culture, respectively, and researchers should keep in mind the impact of long-term culture on the genomic architecture of tumour organoids. It is therefore crucial to cryopreserve early passage organoids and avoid their excessive long-term culture to preserve the genetic features of the original tumours in patient-derived organoids and to ensure experimental reproducibility.

In addition to the use in cancer research, patient-derived organoids have remedied the scarcity of viable and functional human epithelial tissues that are affected by rare heritable monogenic diseases. Intestinal ${ }^{76}$, kidney tubule ${ }^{31}$ and airway ${ }^{16}$ organoids derived from patients with cystic fibrosis showed impaired intraluminal fluid secretion upon activation of cyclic adenosine mono-phosphate and reproduced genotype-specific response to cystic fibrosis transmembrane conductance regulator (CFTR) correctors. Organoid culture of tissues affected by other monogenic disorders, namely microvillus inclusion disease $\mathrm{e}^{77}$ (caused by myocin $\mathrm{Vb}$ or syntaxin 3 deficiency), multiple intestinal atresia ${ }^{78}$ (caused by tetratricopeptide repeat domain 7A deficiency), diacylglycerol-acyltransferase 1 deficiency $^{79}$, Alagille syndrome ${ }^{25}$ (caused by Jagged-1 or notch 2 deficiency) and $\alpha 1$-antitrypsin deficiency ${ }^{25}$, have successfully captured functional, structural, metabolic and developmental errors that characterize these diseases.

Organoids also preserve pathological states that are not necessarily defined by genetic defects. For instance, endometriosis organoids showed intrinsic activation of several oncogenic pathways that paralleled disease progression ${ }^{66}$. Organoids derived from gastric intestinal metaplasia ${ }^{50}$ and Barrett's oesophagus ${ }^{23}$ maintain intestinal marker expression, demonstrating that these intestine-like metaplastic conditions are cell-intrinsically fixed despite the importance of the inflammatory environment in their etiology, that is, Helicobacter pylori infection and chronic acid reflux, respectively. A methylome analysis revealed promoter hypermethylation in gastric intestinal metaplasia organoids and suggested epigenetic modification as a driver of metaplastic transformation ${ }^{50}$. Organoids established from the inflamed tissue of patients with inflammatory bowel disease also preserved characteristic gene expression ${ }^{80,81}$ and DNA methylation patterns that were distinct from those of healthy organoids $^{81}$. Intestinal organoids derived from subjects with coeliac disease showed reduced expression of the genes that are related to the gut barrier function and had increased epithelial permeability ${ }^{82}$. Bile duct organoids established from patients with primary sclerosing cholangitis showed higher expression of immune-activated genes, including downstream targets of interleukin-17A (IL-17A), compared with healthy organoids ${ }^{83}$. Interestingly, a recent study identified specific strains of Klebsiella pneumoniae in the microbiota of patients with primary sclerosing cholangitis. These strains were capable of damaging the surface structure of $2 \mathrm{D}$-cultured human colonic organoids, and this ability was considered to facilitate bacterial translocation and T helper 17 cell response in the host liver ${ }^{84}$. These studies highlight the presence of environmentally imprinted phenotypes in the human epithelium and the validity of using patient-derived organoids in investigating molecular mechanisms, especially epigenetic modifications that underlie such pathological conditions.

Organoid reverse engineering for genetic disease modelling. Although patient-derived organoids have facilitated human disease modelling from a disease- and phenotype-oriented viewpoint, direct establishment of organoids can be hindered in cases where the disease is extremely rare or the genetic defect leads to prenatal lethality. Solidifying the causality between a gene defect and disease phenotypes using genetic perturbation, or the reverse genetics approach, is also pivotal in establishing genotype-phenotype correlations in human genetic disorders. To manipulate genes of interest in human organoids, researchers have implemented conventional gene overexpression and knockdown assays, and, subsequently, clustered regularly interspaced short palindromic repeats (CRISPR)-CRISPR-associated protein (Cas9)-based genome 
a

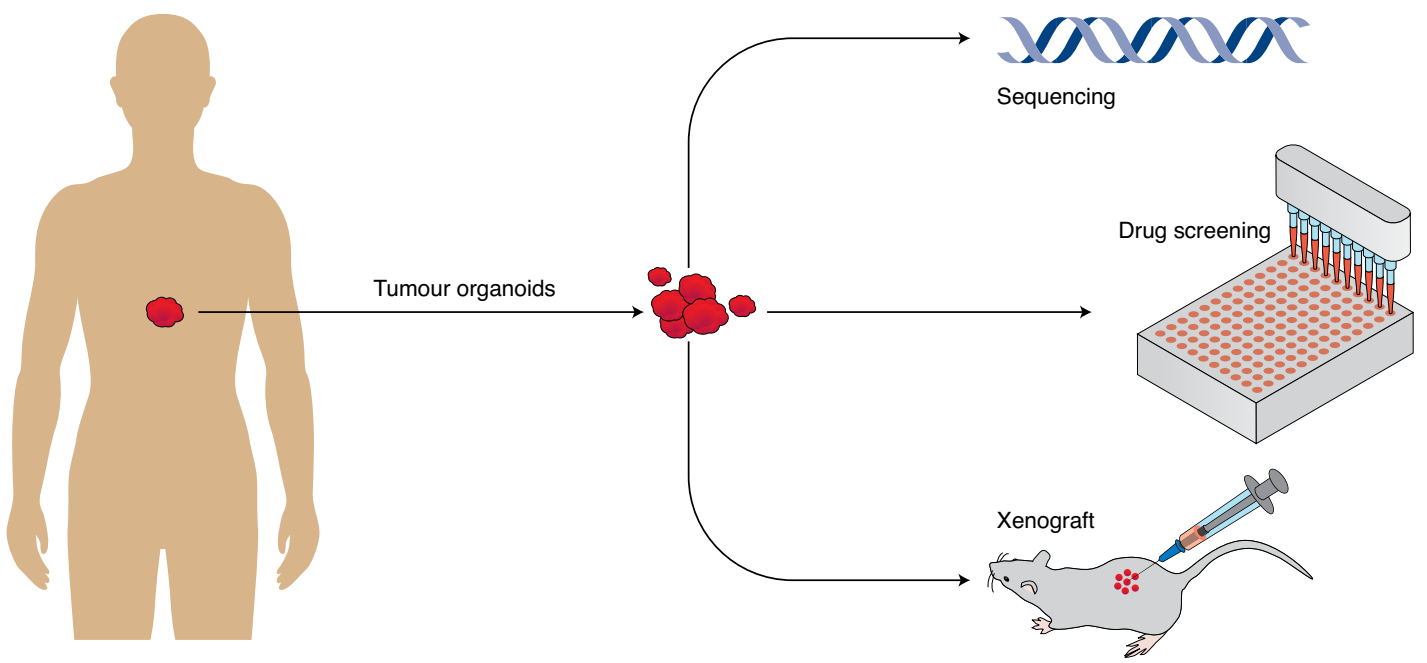

b
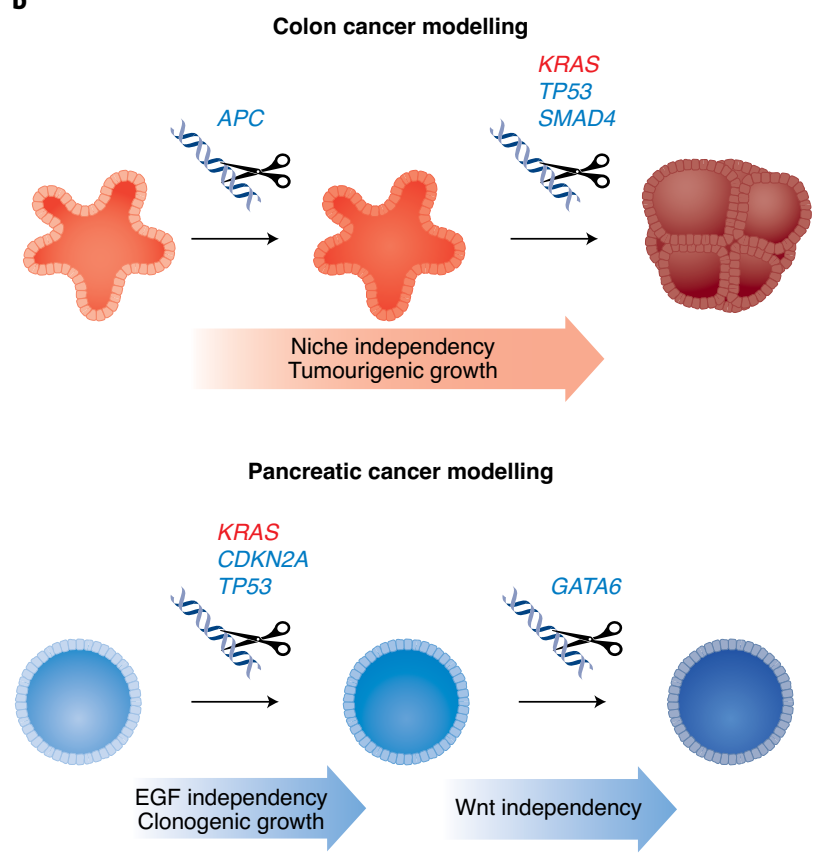

Diffuse-type gastric cancer modelling
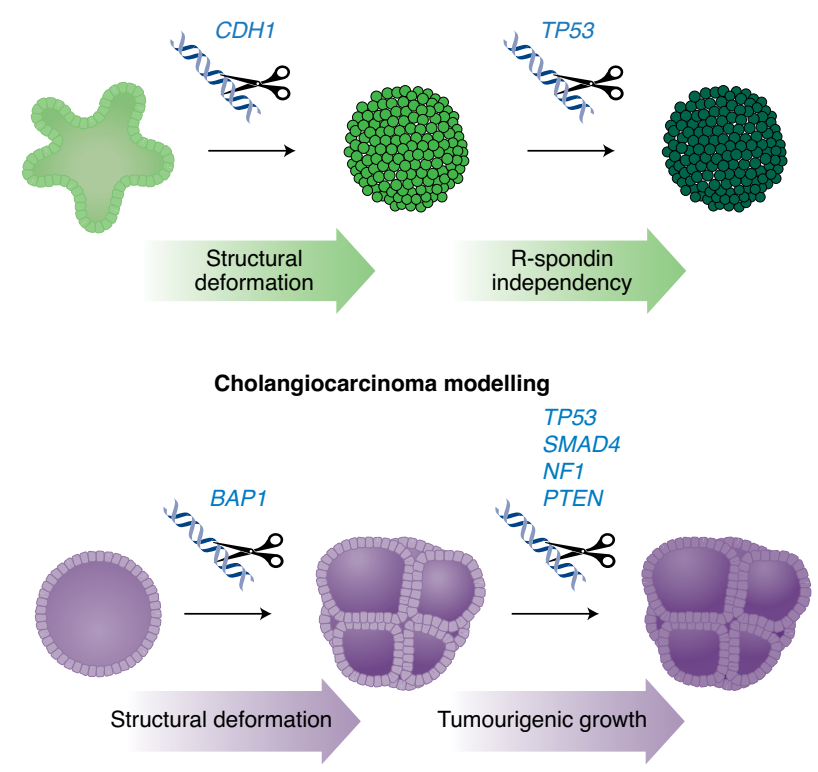

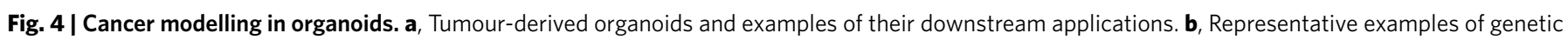
modelling of human cancers using normal organoids and CRSIPR-Cas9-mediated genome editing. Tumour suppressor genes are shown in blue text and the KRAS oncogene is shown in red text.

editing on organoids ${ }^{85}$. Pioneered by the accurate rectification of a defective CFTR sequence in cystic fibrosis organoids ${ }^{86}$, the partnership between CRISPR-Cas9 and organoid technologies has paved a new path for tackling human diseases related to defective gene functions, including monogenic diseases and, for the most part, cancer (Fig. 4b).

Human cancers typically accumulate multiple genomic abnormalities during tumour history. However, whether and how individual genetic flaws derail the cytological behaviour of human tissues has been difficult to address due to the highly complex and heterogeneous nature of genetic defects among human cancers and an experimental hurdle in setting up cancer-relevant mutations in normal human tissues. Initial attempts to adapt CRISPR-Cas9-mediated genome editing of human organoids to functional cancer genomics aimed to genetically reconstitute human colon cancer using the classical genetic model of colorectal cancer progression, or the adenoma-carcinoma sequence, as a genetic blueprint. Sequential disruption of tumour suppressor genes (APC, TP53 and SMAD4) and the introduction of oncogenic hotspot mutations (KRAS and $P I K 3 C A)$ in human normal colonic organoids incremented growth-factor-independent growth in vitro ${ }^{74,87}$ and tumourigenic capacity as xenograft tumours ${ }^{87}$. p53 inactivation also induced chromosomal instability ${ }^{74}$. Independent deletion of $M L H 1^{75}, N T H L 1^{75}$ or $X P C^{88}$, which are related to distinct DNA repair functions, in human normal colonic organoids accelerated the acquisition of unique mutation signatures. Treatment of $B R A F$-mutation-introduced human colonic organoids with TGF- $\beta$ upregulated a set of genes that are related to the epithelial-to-mesenchymal transition ${ }^{89}$. Genetic replication of the molecular chronology of pancreatic ductal cancer using pancreas organoids highlighted the importance of defective GATA-binding factor 6 (GATA6) function in the acquisition of niche-independent growth and disease progression ${ }^{30}$. Knockout of the CDH1 (E-cadherin) gene in human gastric organoids induced structural deformation reminiscent of the diffuse-type 
gastric cancer, and further knockout of TP53 conferred the ability to grow independently of the R-spondin niche ${ }^{50}$. Knockout of $B A P 1$, an epigenetic modifier, distorted epithelial organization and apicobasal polarity in human liver ductal organoids, and additional inactivation of the TP53, SMAD4, NF1 and PTEN genes generated organoids that form xenograft tumours that are histologically comparable to cholangiocarcinoma ${ }^{90}$. Disruption of TP53, PTEN, RB1 and NF1 genes transformed normal human mammary organoids into the luminal-type breast cancer ${ }^{19}$.

In addition to these conservative knockout and knockin experiments, efficient genome cleavage by CRISPR-Cas9 further enabled introduction of complex genomic rearrangements in human colonic organoids and contributed to the genetic modelling of traditional serrated adenoma, a relatively rare subtype of colonic pol$\mathrm{yps}^{91}$. Thus, the strategy to build human tumours from scratch using normal organoids and CRISPR-Cas9 technology allows us to study the pure contribution of defined gene defects to the behaviour of tumour cells and is instructive in decomposing cancer genomics into single genotype-phenotype elements.

Modifying culture conditions to simulate diseased tissue environments. Once organoids are established, researchers are free to arrange the culture substrate and medium composition, just like cell line experiments, for studying the action of epithelial cells in specific environments. Placing organoids in disease-pertinent contexts may perhaps be the simplest approach for disease modelling. For instance, to partially reconstitute the inflammatory milieu in immunological and infectious diseases, previous studies have treated organoids with various cytokines and bacterial products. Stimulation with tumour necrosis factor- $\alpha$ or its combination with interferon- $\gamma$ elicits an apoptotic and necroptotic response in mouse intestinal organoids that genetically lack autophagy related 16 like 1 (ATG16L1, encoded by Atg16l1), a Crohn's disease susceptibility gene in humans, highlighting the protective role of ATG16L1 during epithelial damage by intestinal inflammation ${ }^{92,93}$. ATG16L1 loss also sensitized mouse intestinal organoids to IL-22, which alternatively promotes the regeneration of intestinal stem cells ${ }^{94}$ and protects them from genotoxic stress ${ }^{95}$. Using clonal derivation and gene engineering of organoids, a recent study demonstrated that the colonic epithelium of patients with ulcerative colitis accumulate somatic mutations that provide survival benefit under chronic inflammatory damage induced by IL-17 ${ }^{96}$. These studies effectually used organoids to dissociate the effect of individual cytokines in epithelium- and genotype-specific manners. Inspired by the deposition of type I collagen in the regenerative epithelium following experimental colitis and in the human large intestine affected by ulcerative colitis, another study demonstrated that the remodelling of the ECM that occurs during tissue repair gears the epithelium towards an embryonic state via mechanotransduction and that embedding intestinal organoids into type I collagen gel reproduces this process ${ }^{97}$. Interestingly, parasitic granulomas in the mouse intestine induce a similar developmental reversion in the overlying epithelium through interferon- $\gamma$ signalling ${ }^{98}$, suggesting that ECM properties and inflammatory signalling converge into an identical regenerative fetal phenotype to promote wound repair.

Culture of tumour organoids has also exploited the customizability of the culture composition. By virtue of the extensive compilation of genetic abnormalities that occur in human cancers, we now understand that genetic aberrations in human cancers often hijack stem cell niche pathways ${ }^{99}$, thereby enabling autonomous growth of tumour cells in the absence of the stem cell niche. For instance, human colorectal tumours predominantly acquire $A P C$ mutations, and it follows that most, if not all, colorectal tumour organoids expand in the absence of exogenous Wnt and R-spondin ${ }^{54}$. Similarly, most human pancreatic ductal carcinomas carry KRAS mutations, which enable the EGF-independent growth of patient-derived organoids ${ }^{30}$. Human cancers also acquire other hallmarks of cancer, such as resistance to cell death and hypoxia, through genetically or non-genetically defined mechanisms. These prior knowledges not only allowed us to omit specific growth factors from the culture media but also to efficiently derive tumour organoids from crude tissues by eliminating outgrowing normal organoids and enabling the positive selection of tumour organoids. For instance, treatment with a mouse double minute 2 homologue (MDM2) inhibitor, which activates the intrinsic p53 pathway, and removal of a rho-associated protein kinase (ROCK) inhibitor following single-cell dissociation, which selects organoids that are resistant to anoikis, allowed for efficient derivation of gastric cancer organoids ${ }^{50}$. Some colorectal cancer organoids favoured hypoxic growth, highlighting the importance of preparing several culture patterns with differing oxygen tensions during their establishment ${ }^{54}$. The culture environment with reduced niche activity may briefly represent a barren land to which the tumour cells must acclimate themselves for survival. In a genetically engineered model of pancreatic cancer, placing organoids in a growth-factor-deficient condition was essential for their epigenetic transformation ${ }^{30}$. Specifically, pancreatic cancers adapt to a Wnt-deficient environment through epigenetic silencing of GATA6, which is recurrently observed in a poor prognostic subtype of pancreatic cancer, and genetically engineered organoids gained this phenotype only in the absence of Wnt-3a. Another study demonstrated that mouse colonic organoids with an oncogenic Braf mutation show cell-autonomous activation of the Wnt pathway after a long-term culture ${ }^{100}$. This process accompanied DNA methylation with an aging-like pattern, which suggests that the long-term organoid culture induces a spontaneous remodelling of the epigenetic landscape in a genotype-dependent fashion. Together, exposing organoids to specific cancer-relevant environments is useful not only for facilitating selective expansion of patient-derived tumour organoids but also for modelling tumour progression by inducing epigenetic transformation in vitro.

Organoid co-culture systems for modelling disease ecosystems. In many human diseases, non-epithelial cells often physically or indirectly interact with epithelial cells to modify disease presentations. Probing such interplay between distinct cellular populations can be challenging with stereotypical organoids constituted exclusively of epithelial cells. With the aim of comprehending the cellular diversity engaged in disease ecosystems, previous studies have incorporated non-epithelial cells in the organoid culture system (Fig. 5).

The co-culture system was representatively used for studying the functionality of cancer-associated fibroblast (CAF), a cancer-specific stromal component of the tumour microenvironment that is deemed to have a pleiotropic role in cancer biology. In pancreatic cancer, distinct CAF populations exist, but the controversy as to whether they have tumour suppressive or promotive function has remained unsettled. A recent study demonstrated that mouse pancreatic stellate cells activate an inflammatory programme when co-cultured with mouse tumour organoids and promote organoid growth ${ }^{101}$. Another study showed that a subset of pancreatic CAFs secrete Wnt ligands and that their physical contact with pancreatic cancer organoids supports organoid growth through a juxtacrine molecular communication ${ }^{30}$ (Fig. 5a). Due to the preferential growth of fibroblasts on stiff substrata rather than in soft matrix, this study established tumour-derived organoids and CAFs individually using standard organoid culture and a plastic substrate, respectively. Together, pancreatic CAFs are, at least in the organoid co-culture system, contributory to tumour growth.

In a similar manner, hybrid culture organoids with immune cells has allowed for ex vivo modelling of immuno-epithelial interactions, which include the cross-talk between leukocytes and organoids ${ }^{102-105}$, cytotoxicity of chimeric antigen-engineered 


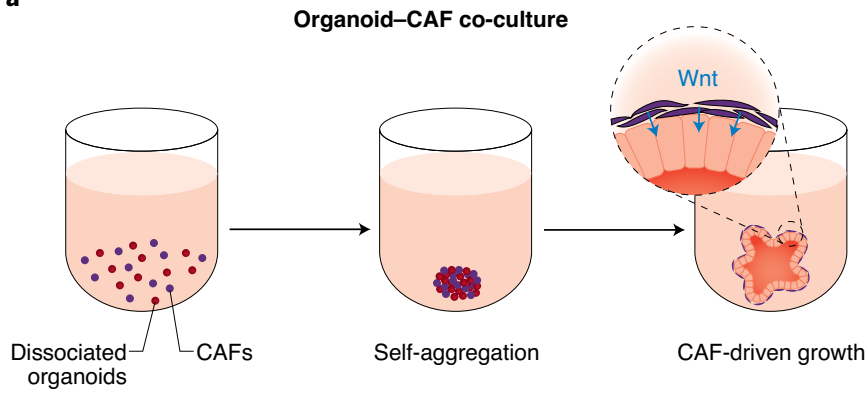

b
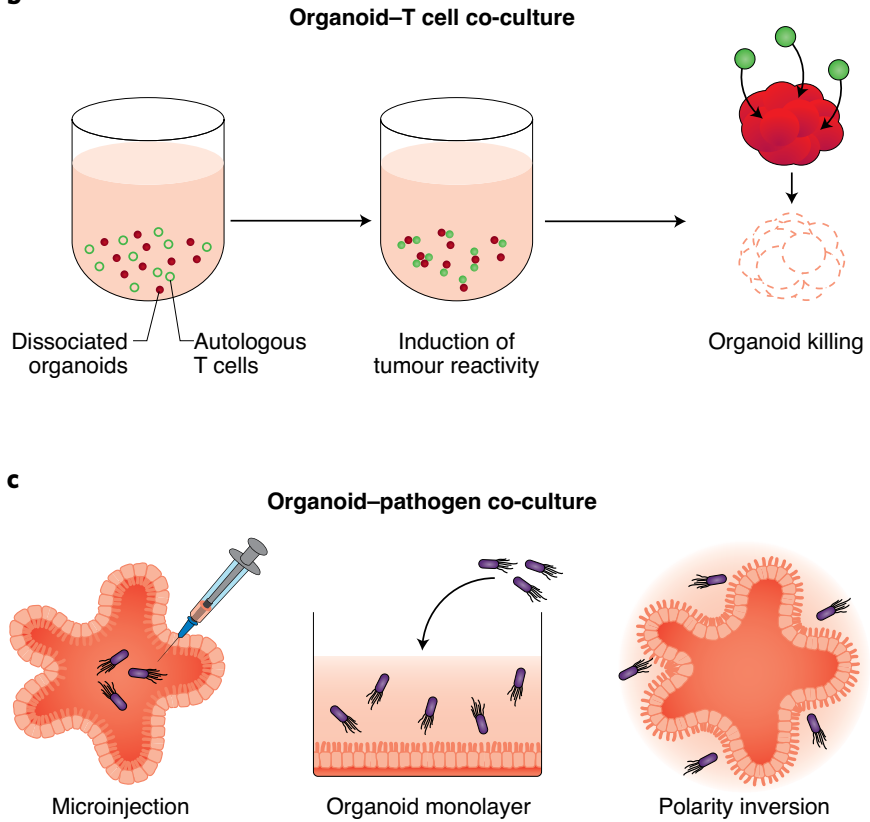

Organoid-pathogen co-culture
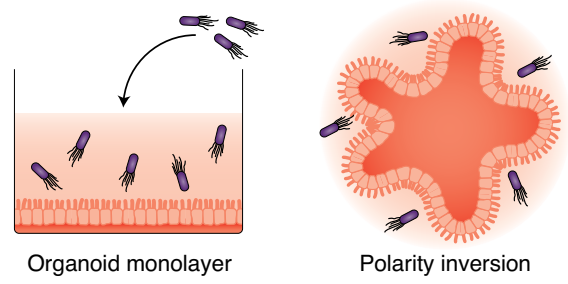

Fig. 5 | Organoid co-culture applications. a, Co-culture of pancreatic cancer organoids with CAFs. A mixture of dissociated organoids and CAFs self-organizes and forms a single CAF-covered organoid. $\mathbf{b}$, An example of immune cell-organoid co-culture. Co-culture of cancer organoids with autologous T cells induces tumour reactivity in T cells. c, Organoid-pathogen co-culture systems and access routes to the lumen side of organoids.

lymphocytes ${ }^{106}$ and tumour education of circulating $\mathrm{T}$ cells by co-culture with autologous tumour organoids ${ }^{107}$ (Fig. 5b). An airliquid-interface-based tumour organoid culture system encompassed the stroma and tumour-infiltrating lymphocytes with preserved T-cell receptor and immunoglobulin repertoires of the parental tumours and recapitulated the programmed cell death protein 1 (PD-1) -dependent immune regulation ${ }^{56}$. These studies collectively demonstrate that increasing the complexity of organoids through the hybrid culture with non-epithelial cells is a pragmatic strategy for studying the interaction between epithelial and stromal cells.

Host-pathogen co-culture as an infection model. Adaptation of the co-culture system further allowed us to study the interplay between epithelial tissues and microorganisms (Fig. $5 \mathrm{c}$ and Table 2 ). Due to the lumen-facing cellular alignment in most 3D organoids, modelling bacterial encroachment that generally occurs at the lumen side of the epithelium has initially relied on the labour-intensive microinjection of bacteria. In parallel to the effort to improve the accuracy and throughput of organoid microinjection using an automated device ${ }^{108}$, the structural and technical limitation of using 3D organoids for studying bacterial infection was largely resolved by the later development of $2 \mathrm{D}$ monolayer organoid culture systems $\mathrm{s}^{109,110}$. Anaerobic culture systems using human intestinal organoids further enabled the conservation of the gut microbiota repertoire, including obligate anaerobic species ${ }^{111,112}$. Inverting the cell polarity of intestinal organoids by means of suspension culture alternatively increased accessibility to the apical side of the organoids and allowed for straightforward organoid-microbe co-culture as well as nutrient transport analysis ${ }^{113}$. These strategies have successfully recreated the interaction between untransformed epithelium and pathogenic bacteria, including Helicobacter pylori ${ }^{22,110,114}$, Salmonella typhimurium ${ }^{38,113,115}$, Listeria monocytogenes ${ }^{113}$, Escherichia coli (enterohemorrhagic ${ }^{109,116}$, enterotoxigenic ${ }^{104}$, enteropathogenic ${ }^{104,109}$ and enteroaggregative $e^{109,117}$ strains), Klebsiella pneumoniae $^{84}$, Clostridium difficile ${ }^{118}$ and chlamydia trachomatis ${ }^{119}$.

As parasites often have complex lifecycles where each phase involves specific host tissues and species, organoids also provide a suitable model for studying parasitic infection. Recent studies infected human intestinal and lung organoids with Cryptosporidium, a parasite that mainly affects the intestinal and respiratory systems, and demonstrated that cryptosporidium can complete its lifecycle in human intestinal organoids ${ }^{120,121}$. Toxoplasma gondii is one of the most common parasites in the human population and has two main lifecycle phases: the sexual stage that is restricted to the cat intestinal epithelium and the asexual stage that can occur in any warm-blooded animals. A study using feline small intestinal organoids as a definitive host identified linoleic acid abundance or a deficiency of delta-6-desturase, an enzyme that is essential for linoleic acid metabolism, as a determinant of sexual reproduction of T. gon$d i i^{39}$, and another study infected bovine and porcine small intestinal organoids with $T$. gondii tachyzoites as a model of invasion to intermediate hosts ${ }^{38}$.

Organoids have further contributed host cells for a range of viruses, such as influenza virus ${ }^{122-124}$, respiratory syncytial virus ${ }^{16}$, human norovirus ${ }^{125}$, human rotavirus ${ }^{126-128}$, enteroviruses ${ }^{129}$, human adenovirus $^{130}$, BK virus ${ }^{31}$, human papillomavirus ${ }^{15}$, herpes simplex virus $^{15}$, human astrovirus ${ }^{131}$ and Middle East respiratory syndrome coronavirus (MERS-CoV) ${ }^{132}$. Of note, some human norovirus strains required bile supplementation for their efficient replication in human small intestinal organoids ${ }^{125}$.

At the time of the preparation of this manuscript, the global health is facing a serious crisis due to the pandemic of coronavirus disease 19 (COVID-19) caused by severe acute respiratory syndrome coronavirus 2 (SARS-CoV-2). As a part of the worldwide effort to break through this situation, human organoids are also utilized for investigating SARS-CoV-2 ${ }^{133-136}$. Such studies may remedy the current insufficiency of our understanding of its infection mode and the mechanism of disease aggravation, and facilitate the discovery of effective drugs.

The strength of organoids in studying infectious disease is particularly evident when specific tissues and cell types are implicated in disease pathophysiology. For instance, goblet cell and enterocyte differentiation in human colon organoids enabled efficient colonization of enterohemorrhagic E. coli ${ }^{116}$. T. gondii parasites were able to undergo sexual reproduction in small intestinal organoids derived from cats, the only mammal known to lack delta-6-desaturase activity in the intestine ${ }^{39}$. Replication of human influenza virus in human airway organoids dramatically increased in the presence of ciliary cells $^{123}$. Human rotavirus infected not only enterocytes but also enteroendocrine cells in human intestinal organoids ${ }^{127,128}$. A specific strain of human adenovirus showed a preferential infection to goblet cells compared with the other cell types in human intestinal $\operatorname{organoids}^{130}$. Genogroup II, genotype 4 (GII.4) human norovirus strains could not efficiently replicate in intestinal organoids derived from individuals that genetically lack functional fucosyltransferase 
Table 2 | List of pathogens used for co-culture with organoids

\begin{tabular}{|c|c|c|}
\hline Pathogen & Host organoid & Refs. \\
\hline \multicolumn{3}{|l|}{ Microbe } \\
\hline Helicobacber pylori & Human stomach & $22,110,114$ \\
\hline \multirow[t]{3}{*}{ Salmonella typhimurium } & $\begin{array}{l}\text { Cow and pig small } \\
\text { intestine }\end{array}$ & 38 \\
\hline & Mouse small intestine & 115 \\
\hline & Human small intestine & 113 \\
\hline Listeria monocytogenes & Human small intestine & 113 \\
\hline \multirow[t]{2}{*}{ Escherichai coli } & Human small intestine & $104,109,117$ \\
\hline & Human large intestine & $109,16,117$ \\
\hline Klebsiela pneumoniae & Human large intestine & 84 \\
\hline Clostridium difficile & Mouse small intestine & 118 \\
\hline Chlamydia trachomatis & Human fallopian tube & 119 \\
\hline \multicolumn{3}{|l|}{ Parasite } \\
\hline \multirow[t]{3}{*}{ Cryptosporidium } & Human small intestine & 120 \\
\hline & Human airway & 120 \\
\hline & Mouse small intestine & 121 \\
\hline \multirow[t]{2}{*}{ Toxoplasma gondii } & $\begin{array}{l}\text { Cat and mouse small } \\
\text { intestine }\end{array}$ & 39 \\
\hline & $\begin{array}{l}\text { Cow and pig small } \\
\text { intestine }\end{array}$ & 38 \\
\hline \multicolumn{3}{|l|}{ Virus } \\
\hline Influenza A virus & Human airway & 122-124 \\
\hline Influenza B virus & Human airway & 124 \\
\hline Respiratory syncytial virus & Human airway & 16 \\
\hline Human norovirus & Human small intestine & 125 \\
\hline Human rotavirus & Human small intestine & $126-128$ \\
\hline Enterovirus & $\begin{array}{l}\text { Human fetal small } \\
\text { intestine }\end{array}$ & 129 \\
\hline Human adenovirus & Human small intestine & 130 \\
\hline BK virus & Human kidney tubule & 31 \\
\hline Human papillomavirus & Human oral mucosa & 15 \\
\hline Herpes simplex virus & Human oral mucosa & 15 \\
\hline Human astrovirus & $\begin{array}{l}\text { Human small and large } \\
\text { intestine }\end{array}$ & 131 \\
\hline MERS CoV & Human large intestine & 132 \\
\hline \multirow[t]{3}{*}{ SARS-CoV-2 } & $\begin{array}{l}\text { Human small and large } \\
\text { intestine }\end{array}$ & 133-135 \\
\hline & Bat small intestine & 135 \\
\hline & Human liver duct & 136 \\
\hline
\end{tabular}

2 (FUT2) and are known to be resistant to human norovirus infection $^{125}$. This genotype-specific phenotype was later confirmed by the genetic manipulation of FUT2 in human intestinal organoids ${ }^{137}$. Such observations have been challenging to make in cell lines and encourages the utilization of the organoid co-culture system for a physiological reconstitution the host-pathogen interface.

Roads and hurdles for clinical implementation of organoids In parallel to the broadening usage of organoids in the exploration of disease biology, preclinical studies capitalizing on organoid technology have portended the deployment of organoids in translational medicine and clinical application. The organoid-based selection of potential responders to cystic fibrosis drugs has spearheaded translation of organoid-based in vitro drug testing to clinical decision-making ${ }^{76,138}$. Beyond monogenic diseases, pilot studies have exhaustively pursued the potential of patient-derived cancer organoids in precision medicine, and these experiments demonstrated variable responses of tumour organoids to anticancer agents and genotype-expected sensitivities to molecular-targeting drugg ${ }^{139}$. Recent proof-of-principle studies further reported that patient-derived tumour organoids can inform radiological response of clinical tumours to chemotherapy $y^{65,140,141}$ and chemo-radiotherapy ${ }^{58,59}$. CRISPR-Cas9-mediated labelling and targeting of cancer stem cells in patient-derived colon cancer organoids illuminated the potential of cancer stem cells targeting as well as the extensive de-differentiation capacity of progenitor cancer cells ${ }^{142}$.

Aside from the use of patient-derived organoids as preclinical disease models, massive expandability and phenotypic reliability of normal tissue-derived organoids justifies their practical usage in regenerative medicine. Human intestinal organoids can repopulate the denuded rectal epithelium of mice and retain self-renewal and multidifferentiation capacities over months as xenografts ${ }^{143}$. Human liver organoids functionally colonize the damaged mouse liver ${ }^{25,26}$. Human bile duct organoids embedded within a collagen tube scaffold can stably bridge the surgical defect in mouse biliary tract ${ }^{27}$. Importantly, none of the organoid xenografts in these models showed a sign of tumourigenesis.

Although such successful transmission of human epithelial structures and functions by organoid transplantation, together with the promise of patient-derived organoids in precision medicine, brightens the prospect of organoid-based assays in clinical practice and in basic research, several obstacles that must be cleared lie on the track. As discussed below, organoids currently bear uncertainties and limitations that are inherent to each of the cellular, extracellular and medium components of the organoid culture system. Recent research is nonetheless beginning to resolve these setbacks by integrating state-of-the-art technologies and material science.

Replacing ECM with synthetic hydrogels. The reliance of organoid culture on natural ECM has been one of the major roadblocks in producing clinical-grade organoids that comply with good manufacturing practice. The de facto standard substrate for organoid culture is the basement membrane matrix (BMM) purified from Englebreth-Holm-Swarm mouse sarcoma, a benign murine tumour that abundantly produces ECM. Although BMM is not the genuine basement membrane that exists as a thin layer and anchors the epithelial lining in normal tissues, the biochemical compatibility of BMM with the native basement membrane has encouraged its usage in $3 \mathrm{D}$ cell culture. Organoid culture favours the growth-factor-reduced variant of BMM to minimize phenotype modulation by undefined growth factors. Type I collagen, which is typically extracted from bovine or porcine tissues, also supports the 3D growth of tissue stem cells ${ }^{97,144}$. Despite the common adoption of these two ECMs in organoid culture, their substitution with non-xenobiotic materials is desirable when considering the usage of organoids in human body.

This growing demand for biosimilar culture substrates has motivated the field to synthesize and bioengineer hydrogels that parallel natural ECMs with respect to the ability to foster the growth and maturation of organoids. Given that biological and physical cues that emanate from the ECM largely influence tissue cell behaviour ${ }^{145}$, the biochemical and mechanical properties of BMM that accepts virtually all types of organoids have been parametric milestones that can be leveraged for increasing layers of bioactivity in synthetic culture scaffolds. The main components of BMM are type IV collagen, laminin, perlecan (heparan sulfate proteoglycan) and nidogen/ entactin, most of which are shown to support the growth of mouse intestinal organoids when individually blended into polyethylene 
glycol hydrogels ${ }^{146}$. The sustained expansion of organoids in type I collagen gel suggests that the biochemical requirement of ECMs in organoid culture is relatively lenient and that BMM constituents are functionally redundant. Polyethylene glycol hydrogels tethered with the arginine-glycine-aspartate (RGD) peptide, the integrin recognition motif found in fibronectin, indeed enabled the survival and growth of mouse and human intestinal organoids ${ }^{146}$. This study further charted the mechanical requirement for organoid growth and demonstrated that stiff ECM is essential for the initial expansion phase of intestinal stem cells, whereas ECM softening by proteolysis promotes subsequent organoid formation and differentiation. Another study found that the incorporation of the $\alpha 2 \beta 1$ integrin affinity motif found in type I collagen allows for enhanced recovery and expansion of human intestinal and endometrial organoids ${ }^{147}$. These are good examples of how fractionalizing the dependency of organoids on BMM and ECM properties can navigate the synthesis of minimal and defined hydrogels for organoid culture.

Although the current efforts are largely dedicated to designing BMM-compatible hydrogels, customizing hydrogels beyond this purpose should provide novel opportunities for approaching ECM-driven phenotypes in organoids, especially from the disease viewpoint. For example, intestinal organoids acquire a fetal and regenerative phenotype in type 1 collagen gel $^{97}$, and simulating this situation using bioengineered synthetic hydrogels may not only provide insights into the role of ECM parameters in the pathophysiology of colitis and tissue fibrosis but also contribute to the prospective production of organoids with enhanced regenerative capacity.

Tunable hydrogels may also shed light onto the ECM-related phenotypes of cancer that are critical for disease progression, namely invasion and metastasis. Although these cancer phenotypes have been extensively investigated from the cellular perspective using genetic perturbation, cell-line-based in vitro experiments, such as the invasion and wound healing assays, and xenograft models, in vitro testing that accurately predicts the invasive and metastagenic capacities of cancer cells in the human body has yet to be established. As reviewed comprehensively elsewhere ${ }^{148}$, tumour cells experience multiple steps en route to the formation of overt metastasis, including invasion, intravasation, circulation, extravasation and colonization, and the competence of tumour cells and the tumour environment both mediate these processes. Some metastatic cancers also show tropism to specific organs with distinct biologi$\mathrm{cal}$ and mechanical properties ${ }^{149}$. Modelling these capabilities on the basis of patient-derived cancer organoids and tailored hydrogels, together with the usage of other technologies such as control-release particles to beacon invasive growth, microfluidics and 3D bioprinting to build the vasculature, and perhaps other types of cells and organoids to recreate the vascular and metastatic niche, will be an exciting strategy for studying cancer biology as well as for generating in vitro preclinical platforms for enhanced precision oncology.

Replacing niche factors with biomimetic compounds. Due to the fixed biological property of BMM and collagen scaffolds, modifying the composition of the culture medium has been the mainstream strategy for establishing organoid culture systems for previously uncultured tissues, for formulating defined medium conditions with improved bioactivity and for studying the environmental impact on organoid behaviour. This approach may be an extension of the historical effort to depart from a serum-supplemented, promiscuous culture condition for cell lines towards a fully defined condition for tissue stem cells. As most of the growth factors, agonists and antagonists supplied in organoid culture derive from recombinant proteins or conditioned medium, replacing them with compounds or other molecules has been a popular approach for creating more defined, bioactive and economical culture conditions. A representative example in this framework is the transitioning usage of Wnt-3a, which is essential for many organoids that are driven by $\mathrm{LGR}^{+}$stem cells.
Wnt ligands require post-translational palmytoylation to become biologically active and are hydrophobic in nature. Recombinant Wnt-3a products therefore include detergents, which hampers their usage at high concentrations and have obliged initial organoid cultures to use serum-supplemented conditioned media for efficient Wnt pathway activation. However, later identification of the afamin protein $^{150}$ and liposome ${ }^{151}$ as stabilizers of Wnt ligands in aqueous solution has realized the stable culture of Wnt-dependent organoids in a serum-free setting. Surrogate Wnt agonists that activate canonical Wnt signalling in a frizzled receptor-selective manner have also been developed ${ }^{152}$. Besides these protein-based refinements, glycogen synthase kinase 3 (GSK-3) inhibition by small molecules renders Wnt activators (both Wnt and R-spondin) dispensable in the culture of mouse intestinal organoids ${ }^{153}$. Of note, GSK-3 inhibitors could not maintain the culture of human intestinal organoids for long term ${ }^{154}$.

Another example of a niche factor that was rationally replaced from the originally reported condition is the p38 MAPK inhibitor, which was essential for efficient EGF receptor pathway activation in human intestinal organoids but interfered with their secretory differentiation $^{23}$. Transcriptome-based inference of active ligandreceptor pairs in the human intestinal stem cell niche, followed by high-throughput growth factor screening on human intestinal organoids, identified that the combination of insulin-like growth factor-1 (IGF-1) and FGF-2 enables efficient propagation of human intestinal organoids in the absence of the p38 inhibitor, while retaining multiple differentiated cell populations ${ }^{24}$. This refined culture method also permitted robust growth of a subset of patient-derived colorectal cancer organoids that showed a reduced growth in the presence of the p38 inhibitor. Although the definitiveness of organoid derivation is a prerequisite for a universal delivery of organoid-guided precision medicine, the establishment rate is highly variable, especially in cancer as noted before. Complementing the permissiveness of the organoid culture environments is therefore imperative, and the displacement of the serum and the p38 inhibitor is only the first step towards this mission. In this regard, organoid research can largely benefit from the recent advance in de novo protein design ${ }^{155}$, and the prospective development of highly active, stable and selective mimics of growth factors and cytokines may present a new avenue for generating minimal but fully defined settings for organoid culture that promote accurate, reproducible and sustainable modelling of tissues and disease.

Enhancing the cyto-biological relevance of organoids. The extent to which an experimental tissue model represents the tissue of origin is a primordial question that should always be revisited when using them as tissue and disease alternatives. For organoids, histological assessment of organoid structure and immunostaining of cardinal maker proteins have visualized how organoids optically and qualitatively resemble native tissues. Electron microscopy has captured the presence of specific cell types and cellular anatomy in organoids at the microstructure level. Digital information, including transcriptome, proteome and epigenome data, has been used as a quantitative readout of the organoid-tissue comparability. These multi-tiered approaches have collectively spotlighted not only the biological authenticity of organoids but also their limitations. Exposure of organoids to highly potent and uniform niche factors orients the organoids towards a proliferative and undifferentiated mode. Overrepresentation of dividing cells in organoids may lead to augmented response to cycle-targeting anticancer drugs, and previous studies indeed have documented discordant response to fluorouracil treatment between patient-derived colorectal cancer organoids and organoid xenografts ${ }^{55}$, as well as clinical tumours ${ }^{141}$. In many cases, human organoids also lack terminally differentiated cells and require medium conversion from expansion to differentiation conditions to produce functional cell types ${ }^{23,25,123}$. Human intestinal 
organoids primarily lack the secretory lineage in an expansion culture medium owing to the activation of the Notch pathway that blocks secretory differentiation ${ }^{23}$. The initial organoid culture of liver tissue propagated cystic structures with ductal features from bipotent ductal progenitors, and treatment with a differentiation medium was required to spawn hepatocytes ${ }^{25}$. Induced differentiation invariably causes growth arrest, and hence phenomena that involve specific cell types, such as hepatocyte-specific production of $\alpha 1$-antitrypsin in liver organoids ${ }^{25}$ and human influenza virus infection in human mucociliary differentiation-facilitated airway organoids ${ }^{123}$, can only be maintained temporarily.

Aiming to offset these experimental burdens, as partly mentioned above, organoid researchers have aspired to strengthen the biological fidelity of organoids by renovating the culture condition. This momentum was further accelerated by the recent rise of single-cell analysis that enables fine-grained molecular characterization of organoids and reference tissues. For instance, substitution of a p38 MAPK inhibitor with IGF-1 and FGF-2 in the culture medium of human intestinal organoids promoted secretory cell differentiation without impairing organoid growth. Single-cell transcriptome analysis clearly illustrated the conserved diversity of differentiated cells, including enteroendocrine cells, paneth cells and microfold cells, in organoids expanded with the refined condition $^{24}$. A recent single-cell census of the human liver demonstrated a transcriptomic difference between epithelial cell adhesion molecule (EPCAM)-positive liver cells and bipotent progenitor-derived liver organoids ${ }^{156}$. However, adjustments to the prior culture condition for liver organoids allowed hepatocytes to form albumin ${ }^{+}$ rosette-like organoids, and single cell-RNA sequencing clearly partitioned hepatocyte liver organoid-derived cells and ductal liver organoid-derived cells ${ }^{26}$. It is thus tempting to investigate how these human hepatocyte organoids compare with human tissues at the cellular level. Single-cell analysis also identified multiple cellular populations that differentially express tubular segment-specific genes in human kidney tubule organoids ${ }^{31}$, and demonstrated cellular heterogeneity and responses to estrogen treatment in human endometrium organoids ${ }^{157}$.

So far, single-cell analysis has been performed for only a handful of human organoids, and further cellular decomposition of organoids derived from other healthy and disease tissues is awaited. For instance, a single-cell atlas of human cancer tissues and their organoids cultured in differing growth factor conditions may provide the optimal culture conditions that enable the organoids to reflect the cellular state of the original tumours, thereby contributing to an enhanced prediction of drug response. High-throughput comparison with counterpart human tissues using single-cell analysis has become a prevailing method to comprehensively survey the maturation, lineage commitment and off-target cells in pluripotent-cell-derived organoids, such as those of brain ${ }^{158}$ and kidney ${ }^{159}$, whereas its application in adult stem-cell-derived organoids is just at the nascent stage. With the current downward trend in the cost for DNA sequencing, systematic and high-definition comparison of organoids and counterpart tissues using single-cell RNA sequencing should be a next standard for benchmarking the accuracy of organoids as a representative of human tissues.

\section{Perspectives and conclusions}

This decade punctuated by the success in the long-term culture of mouse intestinal organoids has perhaps been one of the most kaleidoscopic periods in the chronicle of 3D culture and organoid technology ${ }^{160}$, when researchers have strived to derive organoids from tissues that had been difficult to culture with the existing methods and in parallel have procured diseased organoids mainly as a resource. Now that every end user has gained access to healthy and diseased tissues as organoids and can use this high-fidelity tool for various purposes, the priority in organoid research in the coming decade will be to set up practical stages where organoids can maximally unleash their potentials. As noted above, current organoid models of human diseases are basically tuned for studying either cell-intrinsic or environmental factors that control disease phenotypes. Integrating genetic engineering and effective mimicry of disease environments may enable deeper probing of this duality of human diseases. For example, knockout screening in organoids, followed by co-culture with CAFs, may reveal forward and reverse signalling and ligand-receptor pairs that are operative and actionable in the cancer ecosystem. Genetic screening of organoids and co-culture with a pathogen will identify key mediator and effector molecules that are essential for the host recognition, entry, replication and virulence of the pathogen.

From a more clinically oriented perspective, tissue replacement using gene-corrected organoids holds great promise for restoring vital tissue functions that are impaired by pathogenic genetic variants. Correction of defective CFTR in airway organoids derived from patients with cystic fibrosis ${ }^{161}$ and their transplantation may alleviate respiratory complications. Patients with congenital metabolic diseases that result from an insufficient amount or function of vital enzymes may benefit from transplantation of gene-corrected liver organoids. Realization of such conceptual frameworks can be accelerated by development of efficient delivery methods for CRISPR-Cas9 as well as by advances in CRISPR-based genome editors ${ }^{162}$. Deep profiling of the stem cell niche using spatially resolved single-cell RNA sequencing and single-cell proteomics will also contribute to the development of organoid culture systems with high bioactivity and cytocompatibility, and facilitate production of clinical grade organoids that are ready for transplantation usage.

Although several scientific, technical and ethical ramifications inherent to organoid and stem cell studies have yet to be settled as noted above, we are optimistic about the future of organoid research and believe that, together with advances in other technologies, organoids will continue to open new horizons in biomedical science.

Received: 22 January 2020; Accepted: 30 June 2020;

Published online: 17 August 2020

\section{References}

1. Lancaster, M. A. \& Knoblich, J. A. Organogenesis in a dish: modeling development and disease using organoid technologies. Science 345, 1247125 (2014).

2. Shamir, E. R. \& Ewald, A. J. Three-dimensional organotypic culture experimental models of mammalian biology and disease. Nat. Rev. Mol. Cell Biol. 15, 647-664 (2014).

3. Stelzner, M. et al. A nomenclature for intestinal in vitro cultures. Am. J. Physiol. Gastrointest. Liver Physiol. 302, G1359-G1363 (2012).

4. Date, S. \& Sato, T. Mini-gut organoids: reconstitution of the stem cell niche. Annu. Rev. Cell Dev. Biol. 31, 269-289 (2015).

5. Sato, T. et al. Single Lgr5 stem cells build crypt-villus structures in vitro without a mesenchymal niche. Nature 459, 262-265 (2009).

6. Karthaus, W. R. et al. Identification of multipotent luminal progenitor cells in human prostate organoid cultures. Cell 159, 163-175 (2014).

7. Jarde, T. et al. Wnt and Neuregulin 1/ErbB signalling extends 3D culture of hormone responsive mammary organoids. Nat. Commun. 7, 13207 (2016).

8. Criscimanna, A. et al. Duct cells contribute to regeneration of endocrine and acinar cells following pancreatic damage in adult mice. Gastroenterology 141, 1451-1462 (2011).

9. Huch, M. et al. Unlimited in vitro expansion of adult bi-potent pancreas progenitors through the Lgr5/R-spondin axis. EMBO J. 32, 2708-2721 (2013).

10. Boulter, L. et al. Macrophage-derived Wnt opposes Notch signaling to specify hepatic progenitor cell fate in chronic liver disease. Nat. Med. 18, 572-579 (2012)

11. Huch, M. et al. In vitro expansion of single Lgr5+ liver stem cells induced by Wnt-driven regeneration. Nature 494, 247-250 (2013).

12. Planas-Paz, L. et al. The RSPO-LGR4/5-ZNRF3/RNF43 module controls liver zonation and size. Nat. Cell Biol. 18, 467-479 (2016).

13. Lin, S. et al. Distributed hepatocytes expressing telomerase repopulate the liver in homeostasis and injury. Nature 556, 244-248 (2018). 
14. Clevers, H. Modeling development and disease with organoids. Cell 165, 1586-1597 (2016).

15. Driehuis, E. et al. Oral mucosal organoids as a potential platform for personalized cancer therapy. Cancer Discov. 9, 852-871 (2019).

16. Sachs, N. et al. Long-term expanding human airway organoids for disease modeling. EMBO J. 38, e100300 (2019).

17. Kim, M. et al. Patient-derived lung cancer organoids as in vitro cancer models for therapeutic screening. Nat. Commun. 10, 3991 (2019).

18. Sachs, N. et al. A living biobank of breast cancer organoids captures disease heterogeneity. Cell 172, 373-386 (2018).

19. Dekkers, J. F. et al. Modeling breast cancer using CRISPR/Cas9-mediated engineering of human breast organoids. J. Natl Cancer Inst. 112, 540-544 (2019).

20. Jiang, M. et al. Transitional basal cells at the squamous-columnar junction generate Barrett's oesophagus. Nature 550, 529-533 (2017).

21. Kasagi, Y. et al. The esophageal organoid system reveals functional interplay between Notch and cytokines in reactive epithelial changes. Cell. Mol. Gastroenterol. Hepatol. 5, 333-352 (2018).

22. Bartfeld, S. et al. In vitro expansion of human gastric epithelial stem cells and their responses to bacterial infection. Gastroenterology 148 , 126-136 (2015).

23. Sato, T. et al. Long-term expansion of epithelial organoids from human colon, adenoma, adenocarcinoma, and Barrett's epithelium. Gastroenterology 141, 1762-1772 (2011)

24. Fujii, M. et al. Human intestinal organoids maintain self-renewal capacity and cellular diversity in niche-inspired culture condition. Cell Stem Cell $\mathbf{2 3}$ 787-793 (2018).

25. Huch, M. et al. Long-term culture of genome-stable bipotent stem cells from adult human liver. Cell 160, 299-312 (2015).

26. $\mathrm{Hu}, \mathrm{H}$. et al. Long-term expansion of functional mouse and human hepatocytes as 3D organoids. Cell 175, 1591-1606 (2018).

27. Sampaziotis, F. et al. Reconstruction of the mouse extrahepatic biliary tree using primary human extrahepatic cholangiocyte organoids. Nat. Med. 23, 954-963 (2017).

28. Lugli, N. et al. R-spondin 1 and noggin facilitate expansion of resident stem cells from non-damaged gallbladders. EMBO Rep. 17, 769-779 (2016).

29. Boj, S. F. et al. Organoid models of human and mouse ductal pancreatic cancer. Cell 160, 324-338 (2015).

30. Seino, T. et al. Human pancreatic tumor organoids reveal loss of stem cell niche factor dependence during disease progression. Cell Stem Cell $\mathbf{2 2}$ 454-467 (2018).

31. Schutgens, F. et al. Tubuloids derived from human adult kidney and urine for personalized disease modeling. Nat. Biotechnol. 37, 303-313 (2019).

32. Mullenders, J. et al. Mouse and human urothelial cancer organoids: a tool for bladder cancer research. Proc. Natl Acad. Sci. USA 116, 4567-4574 (2019).

33. Turco, M. Y. et al. Long-term, hormone-responsive organoid cultures of human endometrium in a chemically defined medium. Nat. Cell Biol. 19, 568-577 (2017).

34. Boretto, M. et al. Development of organoids from mouse and human endometrium showing endometrial epithelium physiology and long-term expandability. Development 144, 1775-1786 (2017).

35. Kessler, M. et al. The Notch and Wnt pathways regulate stemness and differentiation in human fallopian tube organoids. Nat. Commun. 6, 8989 (2015).

36. Kopper, O. et al. An organoid platform for ovarian cancer captures intra- and interpatient heterogeneity. Nat. Med. 25, 838-849 (2019).

37. Powell, R. H. \& Behnke, M. S. WRN conditioned media is sufficient for in vitro propagation of intestinal organoids from large farm and small companion animals. Biol. Open 6, 698-705 (2017).

38. Derricott, $\mathrm{H}$. et al. Developing a 3D intestinal epithelium model for livestock species. Cell Tissue Res. 375, 409-424 (2019).

39. Martorelli Di Genova, B., Wilson, S. K., Dubey, J. P. \& Knoll, L. J. Intestinal delta-6-desaturase activity determines host range for Toxoplasma sexual reproduction. PLoS Biol. 17, e3000364 (2019)

40. Post, Y. et al. Snake venom gland organoids. Cell 180, 233-247 (2020)

41. Gao, D. et al. Organoid cultures derived from patients with advanced prostate cancer. Cell 159, 176-187 (2014).

42. Hoffmeyer, K. et al. Wnt/beta-catenin signaling regulates telomerase in stem cells and cancer cells. Science 336, 1549-1554 (2012).

43. Yang, T. B. et al. Mutual reinforcement between telomere capping and canonical Wnt signalling in the intestinal stem cell niche. Nat. Commun. 8, 14766 (2017).

44. Schepers, A. G., Vries, R., van den Born, M., van de Wetering, M. \& Clevers, H. Lgr5 intestinal stem cells have high telomerase activity and randomly segregate their chromosomes. EMBO J. 30, 1104-1109 (2011)

45. Kodack, D. P. et al. Primary patient-derived cancer cells and their potential for personalized cancer patient care. Cell Rep. 21, 3298-3309 (2017).

46. Masters, J. R. Human cancer cell lines: fact and fantasy. Nat. Rev. Mol. Cell Biol. 1, 233-236 (2000)
47. Yu, K. et al. Comprehensive transcriptomic analysis of cell lines as models of primary tumors across 22 tumor types. Nat. Commun. 10, 3574 (2019).

48. Li, X. et al. Organoid cultures recapitulate esophageal adenocarcinoma heterogeneity providing a model for clonality studies and precision therapeutics. Nat. Commun. 9, 2983 (2018)

49. Kijima, T. et al. Three-dimensional organoids reveal therapy resistance of esophageal and oropharyngeal squamous cell carcinoma cells. Cell. Mol. Gastroenterol. Hepatol. 7, 73-91 (2019).

50. Nanki, K. et al. Divergent routes toward Wnt and R-spondin niche independency during human gastric carcinogenesis. Cell 174, 856-869 (2018)

51. Seidlitz, T. et al. Human gastric cancer modelling using organoids. Gut 68, 207-217 (2019).

52. Yan, H. H. N. et al. A comprehensive human gastric cancer organoid biobank captures tumor subtype heterogeneity and enables therapeutic screening. Cell Stem Cell 23, 882-897 (2018).

53. van de Wetering, M. et al. Prospective derivation of a living organoid biobank of colorectal cancer patients. Cell 161, 933-945 (2015).

54. Fujii, M. et al. A colorectal tumor organoid library demonstrates progressive loss of niche factor requirements during tumorigenesis. Cell Stem Cell 18, 827-838 (2016).

55. Schutte, M. et al. Molecular dissection of colorectal cancer in pre-clinical models identifies biomarkers predicting sensitivity to EGFR inhibitors. Nat. Commun. 8, 14262 (2017)

56. Neal, J. T. et al. Organoid modeling of the tumor immune microenvironment. Cell 175, 1972-1988 (2018).

57. Weeber, F. et al. Preserved genetic diversity in organoids cultured from biopsies of human colorectal cancer metastases. Proc. Natl Acad. Sci. USA 112, 13308-13311 (2015).

58. Ganesh, K. et al. A rectal cancer organoid platform to study individual responses to chemoradiation. Nat. Med. 25, 1607-1614 (2019).

59. Yao, Y. et al. Patient-derived organoids predict chemoradiation responses of locally advanced rectal cancer. Cell Stem Cell 26, 17-26 (2019).

60. Broutier, L. et al. Human primary liver cancer-derived organoid cultures for disease modeling and drug screening. Nat. Med. 23, 1424-1435 (2017).

61. Li, L. et al. Human primary liver cancer organoids reveal intratumor and interpatient drug response heterogeneity. JCI Insight 4, e121490 (2019).

62. Nuciforo, S. et al. Organoid models of human liver cancers derived from tumor needle biopsies. Cell Rep. 24, 1363-1376 (2018).

63. Saito, Y. et al. Establishment of patient-derived organoids and drug screening for biliary tract carcinoma. Cell Rep. 27, 1265-1276 (2019).

64. Huang, L. et al. Ductal pancreatic cancer modeling and drug screening using human pluripotent stem cell- and patient-derived tumor organoids. Nat. Med. 21, 1364-1371 (2015)

65. Tiriac, $\mathrm{H}$. et al. Organoid profiling identifies common responders to chemotherapy in pancreatic cancer. Cancer Discov. 8, 1112-1129 (2018).

66. Boretto, M. et al. Patient-derived organoids from endometrial disease capture clinical heterogeneity and are amenable to drug screening. Nat. Cell Biol. 21, 1041-1051 (2019)

67. Hill, S. J. et al. Prediction of DNA repair inhibitor response in shortterm patient-derived ovarian cancer organoids. Cancer Discov. 8, 1404-1421 (2018)

68. Lee, S. H. et al. Tumor evolution and drug response in patient-derived organoid models of bladder cancer. Cell 173, 515-528 (2018).

69. Calandrini, C. et al. An organoid biobank for childhood kidney cancers that captures disease and tissue heterogeneity. Nat. Commun. 11, 1310 (2020).

70. Puca, L. et al. Patient derived organoids to model rare prostate cancer phenotypes. Nat. Commun. 9, 2404 (2018).

71. Tsai, S. et al. Development of primary human pancreatic cancer organoids, matched stromal and immune cells and 3D tumor microenvironment models. BMC Cancer 18, 335 (2018).

72. Roerink, S. F. et al. Intra-tumour diversification in colorectal cancer at the single-cell level. Nature 556, 457-462 (2018).

73. Bolhaqueiro, A. C. F. et al. Ongoing chromosomal instability and karyotype evolution in human colorectal cancer organoids. Nat. Genet. 51 824-834 (2019)

74. Drost, J. et al. Sequential cancer mutations in cultured human intestinal stem cells. Nature 521, 43-47 (2015)

75. Drost, J. et al. Use of CRISPR-modified human stem cell organoids to study the origin of mutational signatures in cancer. Science 358, 234-238 (2017).

76. Dekkers, J. F. et al. A functional CFTR assay using primary cystic fibrosis intestinal organoids. Nat. Med. 19, 939-945 (2013).

77. Wiegerinck, C. L. et al. Loss of syntaxin 3 causes variant microvillus inclusion disease. Gastroenterology 147, 65-68 (2014).

78. Bigorgne, A. E. et al. TTC7A mutations disrupt intestinal epithelial apicobasal polarity. J. Clin. Invest. 124, 328-337 (2014).

79. van Rijn, J. M. et al. Intestinal failure and aberrant lipid metabolism in patients with DGAT1 deficiency. Gastroenterology 155, 130-143 (2018). 
80. Dotti, I. et al. Alterations in the epithelial stem cell compartment could contribute to permanent changes in the mucosa of patients with ulcerative colitis. Gut 66, 2069-2079 (2017)

81. Howell, K. J. et al. DNA methylation and transcription patterns in intestinal epithelial cells from pediatric patients with inflammatory bowel diseases differentiate disease subtypes and associate with outcome. Gastroenterology 154, 585-598 (2018).

82. Freire, R. et al. Human gut derived-organoids provide model to study gluten response and effects of microbiota-derived molecules in celiac disease. Sci. Rep. 9, 7029 (2019).

83. Soroka, C. J. et al. Bile-derived organoids from patients with primary sclerosing cholangitis recapitulate their inflammatory immune profile. Hepatology 70, 871-882 (2019).

84. Nakamoto, N. et al. Gut pathobionts underlie intestinal barrier dysfunction and liver $\mathrm{T}$ helper 17 cell immune response in primary sclerosing cholangitis. Nat. Microbiol. 4, 492-503 (2019).

85. Fujii, M., Clevers, H. \& Sato, T. Modeling human digestive diseases with CRISPR-Cas9-modified organoids. Gastroenterology 156, 562-576 (2019).

86. Schwank, G. et al. Functional repair of CFTR by CRISPR/Cas9 in intestinal stem cell organoids of cystic fibrosis patients. Cell Stem Cell 13, 653-658 (2013).

87. Matano, M. et al. Modeling colorectal cancer using CRISPR-Cas9-mediated engineering of human intestinal organoids. Nat. Med. 21, 256-262 (2015).

88. Jager, M. et al. Deficiency of nucleotide excision repair is associated with mutational signature observed in cancer. Genome Res. 29, 1067-1077 (2019).

89. Fessler, E. et al. TGFbeta signaling directs serrated adenomas to the mesenchymal colorectal cancer subtype. EMBO Mol. Med. 8, 745-760 (2016).

90. Artegiani, B. et al. Probing the tumor suppressor function of BAP1 in CRISPRengineered human liver organoids. Cell Stem Cell 24, 927-943 (2019).

91. Kawasaki, K. et al. Chromosome engineering of human colon-derived organoids to develop a model of traditional serrated adenoma. Gastroenterology 158, 638-651 (2020).

92. Matsuzawa-Ishimoto, Y. et al. Autophagy protein ATG16L1 prevents necroptosis in the intestinal epithelium. J. Exp. Med. 214, 3687-3705 (2017)

93. Pott, J., Kabat, A. M. \& Maloy, K. J. Intestinal epithelial cell autophagy is required to protect against TNF-induced apoptosis during chronic colitis in mice. Cell Host Microbe 23, 191-202 (2018).

94. Lindemans, C. A. et al. Interleukin-22 promotes intestinal-stem-cellmediated epithelial regeneration. Nature 528, 560-564 (2015).

95. Gronke, K. et al. Interleukin-22 protects intestinal stem cells against genotoxic stress. Nature 566, 249-253 (2019).

96. Nanki, K. et al. Somatic inflammatory gene mutations in human ulcerative colitis epithelium. Nature 577, 254-259 (2020)

97. Yui, S. et al. YAP/TAZ-dependent reprogramming of colonic epithelium links ECM remodeling to tissue regeneration. Cell Stem Cell 22, 35-49 (2018).

98. Nusse, Y. M. et al. Parasitic helminths induce fetal-like reversion in the intestinal stem cell niche. Nature 559, 109-113 (2018).

99. Sato, T. \& Clevers, H. Growing self-organizing mini-guts from a single intestinal stem cell: mechanism and applications. Science 340, 1190-1194 (2013)

100. Tao, Y. et al. Aging-like spontaneous epigenetic silencing facilitates Wnt activation, stemness, and Braf ${ }^{\mathrm{V} 600 \mathrm{E}}$-induced tumorigenesis. Cancer Cell 35 , 315-328 (2019).

101. Ohlund, D. et al. Distinct populations of inflammatory fibroblasts and myofibroblasts in pancreatic cancer. I Exp. Med. 214, 579-596 (2017).

102. Nozaki, K. et al. Co-culture with intestinal epithelial organoids allows efficient expansion and motility analysis of intraepithelial lymphocytes. J. Gastroenterol. 51, 206-213 (2016).

103. Rogoz, A., Reis, B. S., Karssemeijer, R. A. \& Mucida, D. A 3-D enteroid-based model to study T-cell and epithelial cell interaction. J. Immunol. Methods 421, 89-95 (2015).

104. Noel, G. et al. A primary human macrophage-enteroid co-culture model to investigate mucosal gut physiology and host-pathogen interactions. Sci. Rep. 7, 45270 (2017).

105. Cook, L. et al. Suppressive and gut-reparative functions of human type $1 \mathrm{~T}$ regulatory cells. Gastroenterology 157, 1584-1598 (2019).

106. Schnalzger, T. E. et al. 3D model for CAR-mediated cytotoxicity using patient-derived colorectal cancer organoids. EMBO J. 38, e100928 (2019).

107. Dijkstra, K. K. et al. Generation of tumor-reactive $t$ cells by co-culture of peripheral blood lymphocytes and tumor organoids. Cell $\mathbf{1 7 4}$ 1586-1598 (2018).

108. Williamson, I. A. et al. A high-throughput organoid microinjection platform to study gastrointestinal microbiota and luminal physiology. Cell. Mol. Gastroenterol. Hepatol. 6, 301-319 (2018).

109. VanDussen, K. L. et al. Development of an enhanced human gastrointestinal epithelial culture system to facilitate patient-based assays. Gut 64, 911-920 (2015).

110. Schlaermann, P. et al. A novel human gastric primary cell culture system for modelling Helicobacter pylori infection in vitro. Gut 65, 202-213 (2016).
111. Jalili-Firoozinezhad, S. et al. A complex human gut microbiome cultured in an anaerobic intestine-on-a-chip. Nat. Biomed. Eng. 3, 520-531 (2019).

112. Sasaki, N. et al. Development of a scalable co-culture system for gut anaerobes and human colon epithelium. Gastroenterology 159, 388-390 (2020).

113. Co, J. Y. et al. Controlling epithelial polarity: a human enteroid model for host-pathogen interactions. Cell Rep. 26, 2509-2520 (2019).

114. Boccellato, F. et al. Polarised epithelial monolayers of the gastric mucosa reveal insights into mucosal homeostasis and defence against infection. Gut 68, 400-413 (2018).

115. Zhang, Y. G., Wu, S., Xia, Y. \& Sun, J. Salmonella-infected crypt-derived intestinal organoid culture system for host-bacterial interactions. Physiol. Rep. 2, e12147 (2014).

116. In, J. et al. Enterohemorrhagic Escherichia coli reduce mucus and intermicrovillar bridges in human stem cell-derived colonoids. Cell. Mol. Gastroenterol. Hepatol. 2, 48-62 (2016).

117. Rajan, A. et al. Novel segment- and host-specific patterns of enteroaggregative Escherichia coli adherence to human intestinal enteroids. MBio 9, e02419-17 (2018).

118. Saavedra, P. H. V. et al. Apoptosis of intestinal epithelial cells restricts Clostridium difficile infection in a model of pseudomembranous colitis. Nat. Commun. 9, 4846 (2018).

119. Kessler, M. et al. Chronic Chlamydia infection in human organoids increases stemness and promotes age-dependent CpG methylation. Nat. Commun. 10, 1194 (2019).

120. Heo, I. et al. Modelling Cryptosporidium infection in human small intestinal and lung organoids. Nat. Microbiol. 3, 814-823 (2018).

121. Wilke, G. et al. A stem-cell-derived platform enables complete Cryptosporidium development in vitro and genetic tractability. Cell Host Microbe 26, 123-134 (2019).

122. Hui, K. P. Y. et al. Tropism, replication competence, and innate immune responses of influenza virus: an analysis of human airway organoids and ex-vivo bronchus cultures. Lancet Respir. Med. 6, 846-854 (2018).

123. Zhou, J. et al. Differentiated human airway organoids to assess infectivity of emerging influenza virus. Proc. Natl Acad. Sci. USA 115, 6822-6827 (2018).

124. Bui, C. H. T. et al. Tropism of influenza B viruses in human respiratory tract explants and airway organoids. Eur. Respir. J. 54, 1900008 (2019).

125. Ettayebi, K. et al. Replication of human noroviruses in stem cell-derived human enteroids. Science 353, 1387-1393 (2016).

126. Yin, Y. et al. Modeling rotavirus infection and antiviral therapy using primary intestinal organoids. Antiviral Res. 123, 120-131 (2015).

127. Saxena, K. et al. Human intestinal enteroids: a new model to study human rotavirus infection, host restriction, and pathophysiology. J. Virol. 90, 43-56 (2016).

128. Chang-Graham, A. L. et al. Human intestinal enteroids with inducible neurogenin-3 expression as a novel model of gut hormone secretion. Cell. Mol. Gastroenterol. Hepatol. 8, 209-229 (2019).

129. Drummond, C. G. et al. Enteroviruses infect human enteroids and induce antiviral signaling in a cell lineage-specific manner. Proc. Natl Acad. Sci. USA 114, 1672-1677 (2017).

130. Holly, M. K. \& Smith, J. G. Adenovirus infection of human enteroids reveals interferon sensitivity and preferential infection of goblet cells. J. Virol. 92, e00250-18 (2018).

131. Kolawole, A. O. et al. Astrovirus replication in human intestinal enteroids reveals multi-cellular tropism and an intricate host innate immune landscape. PLoS Pathog. 15, e1008057 (2019).

132. Zhou, J. et al. Human intestinal tract serves as an alternative infection route for Middle East respiratory syndrome coronavirus. Sci. Adv. 3, eaao4966 (2017).

133. Lamers, M. M. et al. SARS-CoV-2 productively infects human gut enterocytes. Science 369, 50-54 (2020).

134. Zang, R. et al. TMPRSS2 and TMPRSS4 promote SARS-CoV-2 infection of human small intestinal enterocytes. Sci. Immunol. 5, eabc3582 (2020).

135. Zhou, J. et al. Infection of bat and human intestinal organoids by SARS-CoV-2. Nat. Med. 26, 1077-1083 (2020)

136. Zhao, B. et al. Recapitulation of SARS-CoV-2 infection and cholangiocyte damage with human liver ductal organoids. Protein Cell https://doi. org/10.1007/s13238-020-00718-6 (2020).

137. Haga, K. et al. Genetic manipulation of human intestinal enteroids demonstrates the necessity of a functional fucosyltransferase 2 gene for secretor-dependent human norovirus infection. mBio 11, e00251-20 (2020).

138. Berkers, G. et al. Rectal organoids enable personalized treatment of cystic fibrosis. Cell Rep. 26, 1701-1708 (2019).

139. Drost, J. \& Clevers, H. Organoids in cancer research. Nat. Rev. Cancer 18 407-418 (2018).

140. Vlachogiannis, G. et al. Patient-derived organoids model treatment response of metastatic gastrointestinal cancers. Science 359, 920-926 (2018).

141. Ooft, S. N. et al. Patient-derived organoids can predict response to chemotherapy in metastatic colorectal cancer patients. Sci. Transl. Med. 11, eaay2574 (2019) 
142. Shimokawa, M. et al. Visualization and targeting of $\mathrm{LGR}^{+}$human colon cancer stem cells. Nature 545, 187-192 (2017).

143. Sugimoto, S. et al. Reconstruction of the human colon epithelium in vivo. Cell Stem Cell 22, 171-176 (2018).

144. Ootani, A. et al. Sustained in vitro intestinal epithelial culture within a Wnt-dependent stem cell niche. Nat. Med. 15, 701-706 (2009).

145. Watt, F. M. \& Huck, W. T. Role of the extracellular matrix in regulating stem cell fate. Nat. Rev. Mol. Cell Biol. 14, 467-473 (2013).

146. Gjorevski, N. et al. Designer matrices for intestinal stem cell and organoid culture. Nature 539, 560-564 (2016).

147. Hernandez-Gordillo, V. et al. Fully synthetic matrices for in vitro culture of primary human intestinal enteroids and endometrial organoids. Biomaterials 254, 120125 (2020).

148. Lambert, A. W., Pattabiraman, D. R. \& Weinberg, R. A. Emerging biological principles of metastasis. Cell 168, 670-691 (2017).

149. Gao, Y. et al. Metastasis organotropism: redefining the congenial soil. Dev. Cell 49, 375-391 (2019).

150. Mihara, E. et al. Active and water-soluble form of lipidated Wnt protein is maintained by a serum glycoprotein afamin/alpha-albumin. eLife $\mathbf{5}$, e11621 (2016).

151. Tuysuz, N. et al. Lipid-mediated Wnt protein stabilization enables serum-free culture of human organ stem cells. Nat. Commun. 8, 14578 (2017).

152. Janda, C. Y. et al. Surrogate Wnt agonists that phenocopy canonical Wnt and beta-catenin signalling. Nature 545, 234-237 (2017).

153. Yin, X. et al. Niche-independent high-purity cultures of $\operatorname{Lgr}^{+}$intestinal stem cells and their progeny. Nat. Methods 11, 106-112 (2014).

154. Fujii, M., Matano, M., Nanki, K. \& Sato, T. Efficient genetic engineering of human intestinal organoids using electroporation. Nat. Protoc. 10, 1474-1485 (2015).

155. Huang, P. S., Boyken, S. E. \& Baker, D. The coming of age of de novo protein design. Nature 537, 320-327 (2016).
156. Aizarani, N. et al. A human liver cell atlas reveals heterogeneity and epithelial progenitors. Nature 572, 199-204 (2019).

157. Fitzgerald, H. C., Dhakal, P., Behura, S. K., Schust, D. J. \& Spencer, T. E. Self-renewing endometrial epithelial organoids of the human uterus. Proc. Natl Acad. Sci.USA 116, 23132-23142 (2019).

158. Amiri, A. et al. Transcriptome and epigenome landscape of human cortical development modeled in organoids. Science 362, eaat6720 (2018).

159. $\mathrm{Wu}, \mathrm{H}$. et al. Comparative analysis and refinement of human PSC-derived kidney organoid differentiation with single-cell transcriptomics. Cell Stem Cell 23, 869-881 (2018)

160. Simian, M. \& Bissell, M. J. Organoids: a historical perspective of thinking in three dimensions. J. Cell Biol. 216, 31-40 (2017).

161. Vaidyanathan, S. et al. High-efficiency, selection-free gene repair in airway stem cells from cystic fibrosis patients rescues CFTR function in differentiated epithelia. Cell Stem Cell 26, 161-171 (2020).

162. Geurts, M. H. et al. CRISPR-based adenine editors correct nonsense mutations in a cystic fibrosis organoid biobank. Cell Stem Cell 26, 503-510 (2020).

\section{Author contributions}

M.F. and T.S. jointly wrote the manuscript.

\section{Competing interests}

The authors declare no competing interests.

\section{Additional information}

Correspondence should be addressed to M.F. or T.S.

Reprints and permissions information is available at www.nature.com/reprints. Publisher's note Springer Nature remains neutral with regard to jurisdictional claims in published maps and institutional affiliations.

(c) Springer Nature Limited 2020 